\title{
Trypanosoma brucei PolIE suppresses telomere recombination
}

Maiko Tonini ${ }^{1,+}$, , M. A. G. Rabbani ${ }^{1,+}$, Marjia Afrin ${ }^{1}$, and Bibo Li ${ }^{1,2,3,4, *}$

${ }^{1}$ Center for Gene Regulation in Health and Disease, Department of Biological, Geological, and

Environmental Sciences, College of Sciences and Health Professions, Cleveland State

University, 2121 Euclid Avenue, Cleveland, $\mathrm{OH} 44115$

${ }^{2}$ Case Comprehensive Cancer Center, Case Western Reserve University, 10900 Euclid Avenue,

Cleveland, $\mathrm{OH} 44106$, USA

${ }^{3}$ Department of Inflammation and Immunity, Lerner Research Institute, Cleveland Clinic, 9500

Euclid Avenue, Cleveland, $\mathrm{OH} 44195$

${ }^{4}$ Center for RNA Science and Therapeutics, Case Western Reserve University, 10900 Euclid

Avenue, Cleveland, $\mathrm{OH} 44106$, USA

*Correspondence: b.li37@csuohio.edu; (216) 687-2444

†These authors contributed equally

§Current address: Chronic and Airborne Disease Surveillance Coordination, Health Surveillance

Department Ministry of Health, Brasília - Federal District - CEP: 70719-040

Key words: PolIE, telomere recombination, telomeric C-circles, telomere G-overhang,

Trypanosoma brucei 


\begin{abstract}
Telomeres are essential for genome integrity and stability. In T. brucei that causes human African trypanosomiasis, the telomere structure and telomere proteins also influence the virulence of the parasite, as its major surface antigen involved in the host immune evasion is expressed exclusively from loci immediately upstream of the telomere repeats. However, telomere maintenance mechanisms are still unclear except that telomerase-mediated telomere synthesis is a major player. We now identify PolIE as an intrinsic telomere complex component. We find that depletion of PolIE leads to an increased amount of telomere/subtelomere DNA damage, an elevated rate of antigenic variation, and an increased amount of telomere T-circles and C-circles, indicating that PolIE suppresses telomere recombination and helps maintain telomere integrity. In addition, we observe much longer telomere G-rich 3' overhangs in PolIEdepleted cells, which is not dependent on telomerase. Furthermore, the level of telomere DNA synthesis is slightly increased in PolIE-depleted cells, which is dependent on telomerase.

Therefore, we identify PolIE as a major player for telomere maintenance in T. brucei.
\end{abstract}




\section{Introduction}

Telomeres are nucleoprotein complexes at chromosome ends and are essential for genome integrity and chromosome stability $(1,2)$. In most eukaryotic organisms, telomeres consist of simple repetitive sequences with the G-rich strand going 5' to 3' towards the chromosome ends (3). Telomere length maintenance involves several steps, and it is important to coordinate between the telomere G-strand extension and C-strand fill-in. In the S phase, the chromosome internal portion of the telomere is replicated by conventional DNA polymerases, which are incapable of fully replicating the ends of linear DNA molecules, resulting in a so-called "end replication problem" (4). In most eukaryotic organisms, a specialized reverse transcriptase, telomerase, synthesizes the G-rich strand telomere DNA de novo $(5,6)$. Telomerase contains a protein subunit, TERT, which contains the catalytic activity, and an RNA subunit, TR, which provides a short sequence template for telomere synthesis $(5,6)$. Telomerase uses a G-rich single-stranded 3' DNA end as its substrate (7). Hence, the telomere single-stranded 3' G-rich overhang structure is important for telomerase-mediated telomere synthesis (8-11). The telomere G-overhang is also critical for the formation of the telomere T-loop structure $(12,13)$, which in turn helps protect the telomere from illegitimate nucleolytic degradation and DNA damage repair processes (2). Since leading strand DNA synthesis results in blunt-ended DNA products, exonucleases are required to resect these telomere $5^{\prime}$ ends to generate the telomere $3^{\prime}$ G-overhang structure (14). In human cells, the Apollo exonuclease is recruited to the telomere by the duplex telomere DNA binding factor, $\operatorname{TRF} 2(15,16)$, to blunt-ended telomere ends to resect the telomere $5^{\prime}$ ends (17). Subsequently, EXO1 takes over the $5^{\prime}$ to $3^{\prime}$ end degradation process to generate longer telomere G-overhangs (17). After telomerase-mediated telomere Gstrand synthesis, the telomere C-strand is filled-in by the DNA Primase-Polymerase alpha (18, 19). In addition, the coordination between the telomere G-strand synthesis and C-strand fill-in is mainly regulated by proteins binding the single-stranded telomere G-overhang, including POT1 $(20,21)$ and the CST complex (CTC1/STN1/TEN1 in mammals and CDC13/STN1/TEN1 in budding yeast) (22-26). Therefore, the telomere G-overhang can be elongated by telomerase (7) and by exonucleases that degrade the telomere $5^{\prime}$ end sequences (17). On the other hand, it can 
be shortened when the telomere C-strand is filled-in by the DNA Primase-Polymerase alpha (27).

Trypanosoma brucei is a protozoan parasite that causes human African trypanosomiasis. While proliferating in its mammalian host, T. brucei sequentially expresses immunologically distinct VSGs, its major surface antigen, to evade the host immune response. T. brucei has a large VSG gene pool and all VSGs are located at subtelomeric regions (28). However, only those in VSG expression sites (ESs) can be expressed in a strictly monoallelic manner (29). ESs are polycistronic transcription units, and VSG is the last gene in each ES, located immediately upstream of the telomere repeats (30). VSG switching is frequently mediated by DNA recombination and sometimes through transcriptional switches $(31,32)$.

Telomerase-mediated telomere synthesis is the predominant mechanism of telomere maintenance in T. brucei (33-35). T. brucei telomeres also form a T-loop structure (13). In addition, T. brucei telomere has a short 3' single-stranded G-rich overhang $(10,11)$. The telomere structure and telomere proteins are not only essential for T. brucei genome stability and cell proliferation (36) but are also essential for monoallelic VSG expression (37-40) and influence the VSG switching frequency (41-44). However, detailed T. brucei telomere synthesis mechanisms are still not clear. We previously found that T. brucei has very short telomere G-overhangs ( 12 nts) (10) and the telomerase activity is essential for the telomere G-overhang structure (11), suggesting that resection of the telomere $5^{\prime}$ end by an exonuclease, which has not been identified in T. brucei yet, is minimum and/or the telomere C-strand fill-in step is efficient. So far, no protein has been identified to bind specifically to the single-stranded telomere DNA in $T$. brucei, and how telomere C-strand fill-in is achieved is unknown.

In this study, we performed both Proteomics of Isolated Chromatin Segments (PICh) of the telomere chromatin and affinity pull-down of the telomere protein complex. We have identified PolIE as an intrinsic component of the T. brucei telomere complex. We show that PolIE is essential for cell proliferation and helps maintain genome integrity. Importantly, PolIE plays 
critical roles in maintaining telomere stability. First, in cells depleted of PolIE, we detected a much higher amount of telomere T-circles and C-circles, indicating that PolIE normally suppresses telomere homologous recombination (HR). Second, PolIE-depleted cells have much longer telomere G-overhangs, which is not telomerase-dependent. Third, a higher level of telomerase-dependent telomere synthesis is detected in PolIE-depleted cells, indicating that PolIE normally inhibits telomerase-mediated telomere G-strand extension. 


\section{Results}

Identify T. brucei telomere components by PICh and TbTRF/TbTIF2 IP

We previously identified TbTRF as a duplex telomere DNA binding factor (36) and TbTIF2 as a TbTRF interacting factor (42). We have also shown that telomere proteins play important roles in VSG regulation in T. brucei $(37-43,45,46)$. To identify additional telomere proteins, we first pulled down the known telomere protein complex. We tagged both TbTRF and TbTIF2 with FLAG-HA-HA $(\mathrm{F} 2 \mathrm{H})$ at one of their respective endogenous loci and replaced the remaining allele with selectable markers to generate the procyclic form (PF, the insect stage) T. brucei TbTRF $F^{\mathrm{F} H+/-} \mathrm{TbTIF2}{ }^{+\mathrm{F} 2 \mathrm{H} /-}$ strain. The nuclear extracts prepared from this strain and WT cells $\left(\operatorname{TbTRF}^{+/+} \mathrm{TbTIF2}^{+++}\right.$, as a negative control) were first immunoprecipitated (IPed) with the FLAG monoclonal antibody M2 (Sigma) then with the HA monoclonal antibody 12CA5 (MSKCC Antibody \& Bioresource Core Facility), and the final IP products were analyzed by mass spectrometry (Cleveland Clinic Lerner Research Institute Proteomics and Metabolomics Core) (Fig. S1, left). Proteins identified from WT cells were considered as the background noise and were eliminated from those identified in the $T b T R F^{\mathrm{F} 2 \mathrm{H}+-} T b T I F 2^{+\mathrm{F} 2 \mathrm{H} /-}$ cells. To identify all proteins associated with the T. brucei telomere chromatin in an unbiased manner, we also performed Proteomics of Isolated Chromatin segments (PICh) (47). Using an LNA probe containing either the (TTAGGG) 4 sequence or a scrambled sequence (as a negative control), we pulled down the telomere chromatin and analyzed all its protein components by mass spectrometry (Fig. S1, right). Proteins identified using the control probe were considered as the background noise and removed from those identified using the (TTAGGG) 4 LNA probe.

Both IP and PICh identified several hundred proteins, and 283 proteins were identified in both approaches (Fig. 1A). Among these, DNA polymerase IE (Tb927.11.5550) $(48,49)$ is one of the most abundant proteins. In addition, we have identified TbTRF (36), TbTIF2 (42), TbRAP1 (37), NUP98, NUP96, NUP152, SMC1, SMC3, SMC4, PPL2 (48), and TelAP1 (48), all core histones, $\mathrm{H} 3 \mathrm{v}$, and $\mathrm{H} 2 \mathrm{Bv}$, etc. Most of these proteins have been identified to associate with the telomere chromatin previously, confirming that our approaches are successful. 
T. brucei DNA Polymerase IE associates with the telomere chromatin

Although PolIE was previously identified in a pull-down using a telomere oligo and in a TbTRF IP (48), it has not been directly confirmed that PolIE is a telomere protein $(48,49)$. To verify that PolIE is indeed an intrinsic component of the telomere complex, we first C-terminally tagged one POLIE endogenous allele with $13 x$ myc in the bloodstream form $(\mathrm{BF}$, the mammalian infectious stage) T. brucei cells where one TbTIF2 endogenous allele is C-terminally tagged with an F2H epitope (Fig. 1B). POLIE $E^{++}$, POLIE $E^{+m y c /+}, P O L I E^{+/}$, and POLIE $E^{+m y c /}$ cells grew with nearly identical rate (Fig. S2A), indicating that PolIE-myc is functional. In PolIE-myc and TbTIF2-F2H expressing cells, IP of PolIE-myc using the myc monoclonal antibody 9E10 (MSKCC Antibody \& Bioresource Core Facility) pulled down TbTIF2-F2H and TbTRF in addition to PollE-myc (Fig. 1C). Similarly, PolIE-myc and TbTIF2-F2H were present in the TbTRF IP product when a TbTRF rabbit antibody (36) was used (Fig. 1D). Therefore, PolIE interacts with TbTRF and TbTIF2 in $T$. brucei cells.

Subsequently, we performed ChIP experiment using the myc antibody 9 E10 and observed that PolIE-myc, similar to TbTRF, is associated with the telomere chromatin but not the tubulin chromatin (Fig. 2, A, B; (36) Fig. S2B). Furthermore, we performed Immunofluorescent (IF) analysis in PolIE-myc expressing cells using the myc antibody and TbTRF antibody (36), where TbTRF is used as a telomere marker (36). We observed that PolIE-myc is colocalized with TbTRF throughout the cell cycle (Fig. 2C). Therefore, PolIE is an intrinsic component of the telomere complex.

We further investigated whether the association of PolIE with the telomere chromatin depends on TbTRF, which has a duplex telomere DNA binding activity (36). ChIP was performed in PolIE-myc expressing TbTRF RNAi cells before and after induction of TbTRF RNAi for 24 hrs using the myc antibody 9E10, a TbTRF rabbit antibody (as a positive control) (36), or IgG (as a negative control). ChIP products were then hybridized with a telomere and a tubulin probe (as a negative control) (Fig. 2E; Fig. S2, C, D). TbTRF was successfully depleted and the association 
of TbTRF and the telomere chromatin was abolished upon induction of TbTRF RNAi (Fig. 2, D, E; Fig. S2C). However, PolIE still remained at the telomere after depletion of TbTRF (Fig. 2E; Fig. S2, C, D). Therefore, although PolIE and TbTRF interact, PolIE is localized to the telomere independent of TbTRF.

PolIE is essential for cell proliferation and important for cells to cope with DNA damage assaults PolIE is an A type DNA polymerase that has a DNA polymerase domain very similar to that in

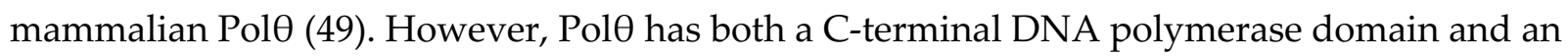
N-terminal helicase domain (50), the latter of which is missing in PollE (49). Biochemical studies

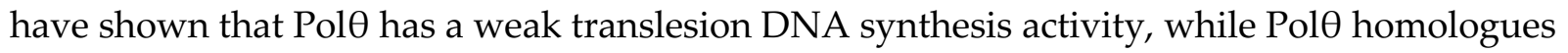
have been shown to be an important factor of Microhomology-Mediated End Joining (MMEJ) that can help repair DNA double strand breaks (DSBs) $(51,52)$. To examine potential functions of PolIE in DNA damage repair, we first established inducible PolIE RNAi strains that also express PolIE-myc from its endogenous locus. Significant depletion of PollE-myc and growth arrest by 24 hrs (Fig. 3, A, B) were observed upon induction of PolIE RNAi, confirming that PolIE is essential for cell proliferation (49).

Mammalian cells lacking PolO are hypersensitive to DNA damaging agents that cause DSBs (50). To examine whether PollE has a similar function in T. brucei, we first examined whether PolIE-depleted cells were more sensitive to EMS. BF PolIE RNAi cells were induced for 0 and 24 hrs followed by treatment with 2 mM EMS (Sigma) for 2 hrs. Subsequently, cells were washed free of doxycycline and EMS and plated in 96-well dishes. We first calculated the plating efficiency (number of survival clones/total number of wells). Relative survival rates (plating efficiency of EMS treated/plating efficiency of untreated) were then calculated for induced and un-induced PolIE RNAi cells. As shown in Fig. S2E, cells depleted of PolIE exhibited a significantly reduced survival rate than un-induced cells. Therefore, PollE-depleted cells are more sensitive to DNA damage reagent EMS, which is consistent with the previous observation that PolIE-depleted cells were slightly more sensitive to MMS treatment (49) 
We further investigated whether depletion of PolIE resulted in hyper sensitivity to UV and cisplatin (causes interstrand crosslink, ICL) in T. brucei. PolIE RNAi cells were induced by doxycycline for $24 \mathrm{hrs}$ and incubated with or without $20 \mu \mathrm{M}$ cisplatin for $1 \mathrm{hr}$ or irradiated with 0,50 mJ, and $100 \mathrm{~mJ}$ of UV light. Subsequently, cells were washed extensively to remove doxycycline and cisplatin and continuously cultured, and cell growth was monitored. As shown in Fig. 3C, cells treated with cisplatin had a poorer relative growth (treated/untreated) in PolIEdepleted cells than in uninduced cells (Fig. 3C, right). Similarly, cells irradiated with UV exhibited much poorer growth than un-irradiated cells (Fig. 3D, left), and the relative growth (irradiated/un-irradiated) is significantly poorer for PollE-depleted cells compared to uninduced cells (Fig. 3D, right). Therefore, depletion of PolIE results in hypersensitivity to both UV irradiation and cisplatin treatment in T. brucei.

Loss of PolIE results in an increased amount of DNA damage at the telomere vicinity and an elevated VSG switching frequency

The above-described observations suggest that PolIE may be important for maintaining telomere integrity. Therefore, we examined whether depletion of PollE caused an increased amount of telomere DNA damage. Western analysis showed that depletion of PolIE by RNAi did not affect the protein levels of TbTRF or TbRAP1 (Fig. 4A). However, we observed an increased amount of $\gamma \mathrm{H} 2 \mathrm{~A}$, a marker of damaged DNA (53), upon depletion of PolIE (Fig. 4A). IF using a $\gamma \mathrm{H} 2 \mathrm{~A}$ rabbit antibody (43) showed that more than $90 \%$ of PolIE-depleted cells exhibited a positive $\gamma \mathrm{H} 2 \mathrm{~A}$ signal, while only $~ 10 \%$ uninduced cells were positive for the $\gamma \mathrm{H} 2 \mathrm{~A}$ signal (Fig. 4B). Subsequently, we performed the ChIP experiment using the $\gamma \mathrm{H} 2 \mathrm{~A}$ antibody and hybridized the ChIP product with telomere and tubulin probes (Fig. 4C). Significantly more $\gamma \mathrm{H} 2 \mathrm{~A}$ was associated with the telomere chromatin after depletion of PolIE, while the same amount of $\gamma \mathrm{H} 2 \mathrm{~A}$ was associated with the tubulin chromatin before and after depletion of PolIE (Fig. 4D). Therefore, depletion of PolIE resulted in an increased amount of DNA damage at the telomere. In addition, we analyzed the $\gamma \mathrm{H} 2 \mathrm{~A}$ ChIP product by quantitative PCR using primers 
specific to the active VSG2, silent VSG16 and mVSG531, the 70 bp repeats in the active ES (70 bp $\mathrm{BES})$, and the $70 \mathrm{bp}$ repeats in a silent ES (70 bp telo) (54). As a control, we also examined whether $\gamma \mathrm{H} 2 \mathrm{~A}$ is associated with rDNA and SNAP50, a chromosome internal gene. As shown in Fig. 4E, depletion of PolIE resulted in an increased amount of $\gamma \mathrm{H} 2 \mathrm{~A}$ associated with the subtelomere chromatin but not with chromosome internal loci.

DSBs at or near the active VSG locus have been shown to be a potent inducer for VSG switching $(55,56)$. Therefore, we further examined whether depletion of PolIE affected VSG switching. We introduced the PolIE RNAi construct into the HSTB261 strain that was established for VSG switching analysis (57) and named the strain as S/IEi, where S stands for switching (42). VSG switching assay was performed as we did previously (41-43). This S strain (HSTB261) has two selectable markers in the active ES: BSD immediately downstream of the ES promotor and PURTK between the $70 \mathrm{bp}$ repeats and the active VSG2 gene (Fig. S3) (57). Switchers will invariably stop expressing the TK gene due to its silencing or its loss and become resistant to Ganciclovir (GCV). Because recovering VSG switchers relies on cell proliferation, we only induced PolIE RNAi for 30 hrs followed by removal of doxycycline from the medium and selection for switchers with GCV. Removal of doxycycline after $30 \mathrm{hrs}$ of induction allowed cells to recover, and these cells were still responsive to doxycycline upon repeated treatment (Fig. 5A). To monitor the PolIE protein level, we tagged one endogenous PolIE allele with 13x myc to generate the S/IEi/PolIE-myc strain. In these cells, induction of PolIE RNAi led to the depletion of PolIE-myc, and removal of doxycycline resulted in the recovery of the PolIE-myc protein level (Fig. 5B, top). Subsequently, in the S/IEi cells, we found that a transient depletion of PolIE for $30 \mathrm{hrs}$ resulted in an $\sim 3$-fold higher VSG switching rate when compared to the S/ev strain with an empty RNAi construct (Fig. 5C), indicating that PolIE suppresses VSG switching. To confirm that this phenotype is specifically due to depletion of PolIE, we introduced an ectopic allele of PolIE-myc in S/IEi cells. Adding doxycycline induced both PolIE RNAi and the expression of the ectopic PolIE-myc (Fig. 5B, bottom). The VSG switching rate in S/IEi+ecPolIEmyc cells is significantly lower than that in S/IEi cells and similar to that in S/ev cells when all 
cells were induced by doxycycline for $30 \mathrm{hrs}$ (Fig. 5C), indicating that the ectopic PollE-myc expression suppressed the increased VSG switching rate phenotype in PolIE-depleted cells.

By examination of the transcription status of the BSD and PUR-TK markers in the originally active ES and by determining whether these markers and the active VSG2 were retained in the switchers, we determined the VSG switching pathways in all obtained switchers (Fig. S3). In the S/ev control cells, a small fraction of the switchers arose from in situ switch (5\%) and crossover (10\%), while VSG gene conversion (43\%) and ES gene conversion/ES loss + in situ events (42\%) were more popular (Fig. 5D; Fig. S3). In contrast, in cells depleted of PolIE, in situ switcher and crossover events were absent, a small fraction of switchers arose from ES gene conversion/ES loss + in situ events (12\%), while VSG gene conversion became the predominant switching events (88\%) (Fig. 5D). Therefore, WT PolIE suppresses VSG gene conversion events by maintaining telomere integrity.

PolIE has been shown to affect VSG silencing (49). To examine PolIE's effect on monoallelic VSG expression, we examined the transcriptomic profile in PollE-depleted cells. Total RNA was isolated from cells where PolIE RNAi was induced for 0 or $30 \mathrm{hrs,} \mathrm{and} \mathrm{poly(A)} \mathrm{RNA} \mathrm{was}$ enriched for making cDNA libraries followed by high throughput RNA sequencing analysis (Novogene Inc.). We found that the expression level of a small number of genes have been changed (either upregulated or downregulated) upon depletion of PollE (Fig. S4A). Among the 68 upregulated genes, 44 are VSGs and 3 are ESAGs (Fig. S4C), indicating that normal VSG silencing is disrupted in cells lacking PolIE. Interestingly, three PARP genes encoding procyclins and transcribed by RNA Pol I were also upregulated (Fig. S4C). Procyclins are the major surface glycoproteins expressed in PF T. brucei that proliferates in the mid-gut of its insect vector and are silent in BF cells. Therefore, PolIE appears to affect the expression of all RNA Pol I transcribed surface antigen genes. To further validate this observation, we did quantitative RT-PCR in cells induced for PolIE RNAi for 24, 30, and 48 hrs. The active VSG2 and rRNA expression level did not change upon depletion of PolIE, while the expression levels of all tested 
silent VSGs (including ES-linked VSGs 3, 6, 9 and two metacyclic VSGs) were upregulated for nearly 10 folds after a 30-hr induction of PolIE RNAi (Fig. S4B).

To further confirm that depletion of PolIE resulted in a true VSG derepression phenotype and that different originally silent VSGs were derepressed simultaneously in individual cells, we performed IF. As shown in Fig. S4D, the originally silent VSG6 and VSG3 were both expressed after PolIE was depleted. VSG6 was more predominantly deposited on the cell surface, while VSG3 was mostly in the cytoplasm. Nevertheless, co-expression of originally silent VSGs strongly suggests that this is a true VSG derepression phenotype. Depletion of PolIE also resulted in downregulation of a number of genes (Fig. S4A), and more than one third of the affected genes are involved in metabolism (Fig. S4C). However, downregulated genes are much more mildly affected than the up-regulated genes (Fig. S4A).

\section{PolIE-depleted cells have an increased amount of T-circles and C-circles}

We found that depletion of PolIE led to an increased number of VSG gene conversion events. We suspect that depletion of PolIE also affects telomere stability. T. brucei does not have the Non-Homologous End Joining (NHEJ) machinery (58), but HR frequently occurs at the telomere vicinity (e.g. mediating VSG switching), and intratelomeric HR can result in extra chromosomal telomere circles (T-circles, Fig. S5A, left), which frequently have nicks on both DNA strands. T. brucei telomere DNA also forms the T-loop structure (13), and excision of T-loop can also form T-circles (59) (Fig. S5A, left). We performed 2D gel electrophoresis, which separates circular from linear DNA molecules more clearly than 1D gel electrophoresis, followed by Southern hybridization with a telomere probe. In PolIE RNAi cells, before the induction of PolIE RNAi, there is a faint signal representing the circular DNA (Fig. 6, A, B). After depletion of PolIE, the T-circle signal is much stronger (Fig. 6, A, C), indicating that PollE suppresses T-circle formation. We further performed the $\varphi 29$ DNA polymerase-mediated telomere C-circle assay (Fig. S5, A, B) (60), which can only amplify circles with nicks/gaps on one strand. Strikingly, depletion of PolIE led to a significant increase in the amount of telomere C-circles but did not 
affect the telomere G-circles (Fig. 6, D, E; Fig. S5C). These observations suggest that PolIE normally suppresses telomere HR.

The telomere C-circle has been identified as a hallmark of ALT cancer cells (61), which maintain the telomere length through telomere HR rather than by telomerase (62). In addition, inhibiting telomerase activity can induce cancer cells to activate the ALT telomere maintenance pathway (63). Therefore, we examined whether deleting the telomerase RNA gene (TbTR) could enhance the increased telomere C-circle phenotype in PolIE-depleted cells. Interestingly, there is a much lower level of telomere C-circles in TbTR null cells than in WT cells (Fig. 6, D, E). This is also confirmed when comparing uninduced PolIE RNAi and TbTR null/PolIE RNAi cells: deleting TbTR decreased the telomere C-circle amount significantly (Fig. 6E). Therefore, deleting telomerase does not induce telomere HR directly, at least within a short time frame. In addition, depletion of PolIE in the TbTR null background again induced a significant increase in the telomere C-circle level (Fig. 6, D, E), indicating that the increased amount of telomere C-circles in PollE-depleted cells does not depend on telomerase.

\section{Depletion of PolIE results in an increased amount of telomere G-overhangs}

The active ES-adjacent telomere is transcribed by RNA Pol I into a long non-coding telomere repeat containing RNA (TERRA) $(43,46,64)$. Together with the telomere DNA, TERRA has a propensity to form the telomeric R-loop (65), a higher than WT level of which has been shown to induce more frequent telomere/subtelomere recombination in T. brucei $(43,46)$. Since PolIE depletion induced a mild VSG derepression (Fig. S4), we suspect that depletion of PolIE may also result in a higher level of TERRA. To our surprise, we detected a lower level of TERRA in PolIE RNAi cells 24 hrs after than before the induction in both northern hybridization and slot blot analyses (Fig. 7). Therefore, it is unlikely that the increased telomere/subtelomere recombination is caused by an elevated telomeric R-loop level. 
Telomere HR can also be induced by long telomere G-overhangs, which can initiate strand invasion more efficiently. Therefore, we tested whether depletion of PolIE affected the telomere G-overhang structure in T. brucei. WT T. brucei cells have short telomere G-overhangs that are estimated to be 12-nt long or shorter $(10,11)$. Indeed, we only detected a very faint telomere Goverhang signal in WT cells using the native in-gel hybridization analysis (Fig. 8A). However, upon depletion of PollE, we observed 20-fold more intensive telomere G-overhang signal (Fig. 8, A, C). This signal was sensitive to Exo I treatment, which degrades 3' end single-stranded DNA specifically (Fig. 8A), indicating that the G-rich single-stranded telomere DNA is located at the end of the chromosome. In addition, only the C-rich TelC probe [(CCCTAA) 4 detected telomere G-rich overhang signals (Fig. 8A), while the TelG probe [(TTAGGG)4] did not yield detectable signals (Fig. 8B), indicating that the telomere single-stranded DNA has a G-rich sequence. We also performed Pulsed-Field Gel Electrophoresis (PFGE) to separate intact T. brucei chromosomes and performed the same native in-gel hybridization analysis. Only G-rich telomere overhang signal was detected, and PolIE depletion greatly enhanced the telomere Goverhang signal (Fig. S6), confirming the results shown in Fig. 8. Our observations suggest that PolIE normally suppresses the telomere HR by limiting the length of telomere G-overhang. In addition, the EtBr-stained gel and the post-denaturation hybridization result showed more smeary DNA species in PolIE-depleted cells than in WT or uninduced PolIE RNAi cells (Fig. S6, left and right), further indicating that depletion of PollE leads to an increased amount of genomic DNA degradation.

T. brucei telomeres have a short 3' G-overhang, and the telomerase activity is a predominant factor for the telomere G-overhang length maintenance $(10,11)$. We further examined the telomere G-overhang level in TbTR null/PolIE RNAi cells to determine whether the increased amount of telomere G-overhang in PolIE-depleted cells depends on the telomerase activity. Before induction of PolIE RNAi, the telomere G-overhang signal in TbTR null/PolIE RNAi cells was undetectable (Fig. 8D, left). However, a significantly higher amount of the telomere Goverhang signal was observed in TbTR null/PolIE RNAi cells 24 hrs after depletion of PolIE (Fig. $8 \mathrm{D}$, left), indicating that the longer telomere G-overhang phenotype in PolIE-depleted cells is 
not dependent on the telomerase activity. In addition, quantification of the hybridization signals indicated that depletion of PolIE in the TbTR null background caused $\sim 13$-fold increase in the telomere G-overhang level, which is similar to that ( 12-fold increase) in the WT TbTR background. As expected, no TelG hybridization signal was detected in TbTR null/PolIE RNAi cells before and after induction.

The subtle increase in telomere DNA synthesis in PolIE-depleted cells is telomerase-dependent Mammalian ALT cancer cells experience frequent telomere DNA breaks that lead to elevated levels of telomere G-overhang, telomere C-circles, and telomere HR (66). We observed that PolIE depletion led to increased telomere DNA damage, telomere G-overhang length, and telomere C-circle level. Therefore, we further investigated whether PolIE is essential for telomere maintenance. Genomic DNA was isolated from PolIE RNAi cells at 0 and 24 hrs after induction and digested with $\mathrm{MboI}$ and AluI followed by electrophoresis on an agarose gel and subsequent Southern hybridization using a telomere repeat probe (67). Within $24 \mathrm{hrs}$ of PolIE RNAi induction, no telomere length change was observed (Fig. 9A).

The telomere length changes in PolIE-depleted cells may be slow, and telomere Southern may not be sensitive enough to detect subtle changes within a short time frame. Therefore, we examined telomere DNA synthesis using an EdU labeling technique. PolIE depletion leads to cell growth arrest, which prevents proper incorporation of EdU during DNA synthesis. Hence, we performed the EdU labeling after a very brief induction of PolIE RNAi, before the cells have entered into a full growth arrest. PolIE RNAi cells were induced for 0 or 12 hrs before the cells were labeled with EdU for $3 \mathrm{hrs}$. Total genomic DNA was isolated followed by the CLICK reaction so that EdU-labeled DNA was conjugated to desthiobiotin and subsequently pulled down by streptavidin beads. The resulting EdU-labeled DNA was then hybridized with a telomere probe, and the hybridization intensities were quantified. To our surprise, telomeric DNA synthesis was at a mildly higher level in PolIE-depleted cells than in un-induced cells, while tubulin DNA replication remained the same (Fig. 9B). 
Telomerase-mediated telomere synthesis is the major mechanism of telomere maintenance in $T$. brucei (33-35). To further investigate whether the subtly increased telomere DNA synthesis is dependent on telomerase, we performed the EdU labeling experiment in TbTR null/PolIE RNAi cells. In the telomerase null background, depletion of PolIE no longer caused any increase in the level of telomere DNA synthesis (Fig. 9C), indicating that the subtle increase in the telomere DNA synthesis observed in PolIE-depleted cells is telomerase-dependent. 


\section{Discussion}

We identified many proteins that associate with the telomere chromatin by PICh, which is a powerful approach to isolate locus-specific chromatin (47). More than 280 proteins have been identified in both PICh and the TbTRF/TbTIF2 protein complex, confirming that this first application of PICh in T. brucei is successful and can be further applied to identify proteins that associate with other loci with repetitive sequences in T. brucei.

Although PolIE was originally identified as a protein that can bind to a TTAGGG repeat containing DNA oligo (48), it was not confirmed that PolIE is a telomere chromatin component. The fact that PolIE was identified in both PICh and as a component of the TbTRF/TbTIF2 protein complex strongly suggests that PolIE is a telomere protein. Our PolIE ChIP result further verifies that PolIE indeed associates with the telomere chromatin. In addition, we observe that PolIE co-localizes with TbTRF, a good telomere marker (36), further indicating that PolIE is a telomere protein. Interestingly, although PolIE belongs to the DNA Polymerase I family in $T$. brucei (49), it has similar DNA crosslink repair functions as mammalian A-family DNA polymerase PolO and POLN. Similar to PolIE, human DNA polymerase $v$ (POLN) has only the C-terminal DNA polymerase domain (68), and POLN helps repair DNA crosslinks $(69,70)$. In addition, Pol $\theta$, having both the C-terminal DNA polymerase domain and the N-terminal helicase domain, is also important for repairing UV-induced DNA damage in skin (71) and POLQ (encoding Pol $\theta$ ) null mouse is hypersensitive to ICL $(72,73)$. The function and domain structure similarities between PolIE and mammalian POLN suggest that PolIE is more homologous to POLN. Nevertheless, PolIE-depleted cells are also mildly more sensitive to EMS and MMS treatments (Fig. S2E) (49), indicating that PolIE is important for maintaining genome integrity.

We found that PolIE plays a critical role in maintaining telomere stability. Several observations indicate that PolIE suppresses HR at the telomere vicinity. First, more telomere T-circles can be detected in PolIE-depleted cells than in WT cells. Second, PollE-depletion leads to an increased amount of telomere C-circles. Third, a transient depletion of PolIE results in many more VSG 
gene conversion-mediated VSG switching events. Therefore, PolIE clearly helps maintain telomere stability by suppressing telomere HR. Mammalian PolO has also been shown to promote alternative NHEJ (MMEJ) at dysfunctional telomeres and translocations at nontelomeric genome loci but suppresses HR-mediated DNA damage repair (52). On the other hand, mammalian POLN does not seem to have any telomere-specific functions. In this sense, PolIE appears to have functions more similar to the mammalian PolO.

Most strikingly, we discover that PolIE plays a critical role in maintaining a proper telomere 3' G-rich overhang structure. Depletion of PolIE greatly increases the telomere 3' G-rich overhang length, suggesting that PolIE regulates factor(s) contributing to the telomere G-overhang structure, including the telomerase-mediated G-strand synthesis, the C-strand fill-in, and the 5' telomere end resection by exonuclease(s) (27). We previously showed that WT T. brucei cells have very short telomere G-overhangs ( $12 \mathrm{nts})(10)$. In addition, in telomerase null T. brucei cells, the telomere 3' G-overhang length is significantly diminished (11). Interestingly, depletion of PolIE leads to a higher level of the telomerase-mediated telomere synthesis, indicating that PolIE normally suppresses the action of telomerase at the telomere. The telomerase-mediated telomere G-strand extension no doubt contributes to the longer telomere G-overhangs observed in PolIE-depleted cells. However, this does not seem to be the only contributing factor. In the TbTR null background, the telomere G-overhangs are much longer after PolIE depletion, indicating that this phenotype is not completely telomerase-dependent. However, whether PolIE affects telomere C-strand fill-in and/or 5' telomere resection will need further investigation.

Interestingly, PolIE appears to maintain telomere stability using different mechanisms than TbRAP1 and TbTRF. Both TbRAP1 and TbTRF suppress the TERRA and the telomeric R-loop levels, which in turn helps maintain telomere integrity and stability $(43,46)$. However, we find that depletion of PolIE results in a lower level of TERRA. It is possible that the long telomere Goverhangs in PolIE-depleted cells form a G-quadruplex structure, which blocks transcription 
elongation of RNA Pol I and results in a lower level of TERRA transcription. However, more experiments will be necessary to test this hypothesis.

T. brucei does not have any NHEJ mechanism (58), but telomere/subtelomere HR is a realistic threat to telomere stability, as HR-mediated VSG switching is frequently observed as a major VSG switching mechanism (41-44, 55-57, 74-76). Therefore, it is significant to discover that $T$. brucei has multiple mechanisms to suppress telomere HR, mediated by various telomere proteins including TbRAP1 (43), TbTRF (46), and PolIE. 


\section{Materials and Methods}

\section{$\underline{\text { T. brucei strains }}$}

Procyclic form (PF, at the insect stage) T. brucei WT cells (Lister 427) were transfected with pSKTbTRF-ko-Hyg (36) and pSK-TbTIF2-ko-BSD (42) to delete one allele of TbTRF and TbTIF2, respectively. The resulting cells were transfected with SacII digested pSK-F2H-TbTRF-Pur-tar and pSK-TbTIF2-F2H-Phleo-tar (42) to insert the FLAG-HA-HA (F2H) tag to the N-terminus of the remaining TbTRF allele and to the C-terminus of the remaining TbTIF2, respectively. The resulting TbTRF ${ }^{\mathrm{F} 2 \mathrm{H}+/}$ TbTIF2+F2H/- cells were used for the 2-step IP of the TbTRF/TbTIF2 protein complex.

All bloodstream form (BF) T. brucei strains used in this study were derived from VSG2expressing Lister 427 cells that express the T7 polymerase and the Tet repressor (Single Marker, aka SM) (77). The HSTB261 strain is derived from SM and specifically designed for VSG switching assay (57), which we renamed as the S strain for easier reference (42). SM cells, $\mathrm{TbTIF}^{+\mathrm{F} 2 \mathrm{H} /+}(42)$, and the S cells were transfected with pSK-PolIE-myc13-Hyg-tar to tag one endogenous allele of PolIE with a C-terminal tag including 13 repeats of myc. The SM cells expressing PolIE-Myc from one of its endogenous loci $\left(\right.$ PolIE $\left.^{+m y c /+}\right)$ were transfected with pZJM $\beta$ TbTRF (36) and pZJM $\beta$-PolIE to generate inducible TbTRF RNAi and PolIE RNAi strains, respectively. The $S$ and S/PolIE-myc cells were transfected with pZJM $\beta$-PolIE to generate S/IEi and S/IEi/PolIE-myc strains. S/IEi cells were transfected with pLew100v5-PolIE-myc to generate the S/IEi + ecPolIE-myc strain. TbTR ${ }^{--}$cells (34) were transfected with pZJM $\beta$-PolIE to generate the $\mathrm{TbTR}^{-/}$PolIE RNAi strain. SM and PolIE ${ }^{+\mathrm{myc} /+}$ cells were transfected with pSK-PolIE-ko-BSD to generate PolIE ${ }^{+-}$and PolIE ${ }^{+m y c /-}$ cells.

\section{$\underline{\text { Plasmids }}$}

A 500 bp genomic DNA fragment upstream of the TbTRF gene, the Puromycin resistance gene (PUR), the $\alpha / \beta$ tubulin intergenic sequence, the F2H-tag, and a $500 \mathrm{bp}$ fragment of the TbTRF 
gene (encoding the N-terminus) are inserted into pBluescript SK in this order to generate pSKF2H-TbTRF-Pur-tar.

A $400 \mathrm{bp}$ PolIE ORF fragment (at the C-terminus), a myc13 tag, the $\alpha / \beta$ tubulin intergenic sequence, the Hygromycin resistance gene (HYG), and a 500 bp genomic DNA fragment downstream of the PolIE gene were inserted into pBluescript SK in this order to generate pSKPolIE-myc13-Hyg-tar.

A 470 bp DNA fragment at the N-terminus of PolIE ORF and a 520 bp DNA fragment at the Cterminus of PolIE ORF were inserted into pZJM $\beta$ (78) in tandem to generate pZJM $\beta$-PolIE RNAi construct.

The Blasticidin-resistance gene (BSD) flanked by genomic DNA fragments upstream and downstream of the PolIE gene, respectively were inserted into pBluescript SK to make pSKPolIE-ko-BSD.

Nuclear Fractionation and 2-Step Immunoprecipitation

$5 \times 10^{10} \mathrm{TbTRF}^{\mathrm{F} 2 \mathrm{H}+/} \mathrm{TbTIF2} 2^{\mathrm{F} 2 \mathrm{H} /-}$ PF cells were harvested by centrifugation followed by snap freezing in liquid nitrogen. After thawing cells, the cell pellet was resuspended in the hypotonic buffer (10 mM HEPES pH 7.9; 10 mM KCl; 2.5 mM MgCl2; 1 mM EDTA; 1 mM DTT; Complete protease (Roche), $1 \mathrm{mM}$ PMSF, $1 \mu \mathrm{g} / \mathrm{ml}$ Leupeptin, $0.5 \mathrm{mg} / \mathrm{ml}$ TLCK, and $1 \mu \mathrm{g} / \mathrm{ml}$ Pesptatin A) and incubated on ice for 10 minutes, followed by adding NP-40 to a final concentration of $0.2 \%$. Cells were homogenized in a glass douncer until $\sim 80 \%$ of the cells were broken. The lysate was overlaid on top of $0.3 \mathrm{~mL}$ of sucrose buffer ( $0.8 \mathrm{M}$ sucrose in hypotonic buffer) and centrifuged at $7 \mathrm{krpm}$ for 10 minutes at $4^{\circ} \mathrm{C}$. After removing the top cytosolic and sucrose layers, the nuclear fraction was resuspended in the TBS buffer $(50 \mathrm{mM}$ Tris $\bullet \mathrm{HCl}, \mathrm{pH} 7.4 ; 420 \mathrm{mM} \mathrm{NaCl}$; Complete protease (Roche), $1 \mathrm{mM}$ PMSF, $1 \mu \mathrm{g} / \mathrm{ml}$ Leupeptin, $0.5 \mathrm{mg} / \mathrm{ml}$ TLCK, and $1 \mu \mathrm{g} / \mathrm{ml}$ Pesptatin A) and digested with Benzonase on ice for 20 minutes followed by centrifugation at 13 
krpm for 10 minutes at $4^{\circ} \mathrm{C}$. The cell lysate was then diluted with $50 \mathrm{mM}$ Tris- $\mathrm{HCl}, \mathrm{pH} 7.4$ so that the final concentration of $\mathrm{NaCl}$ reaches $150 \mathrm{mM}$. The cell lysate was pre-cleared with Dynabeads Protein G (ThermoFisher) then incubated with Anti-FLAG M2 magnetic beads (Sigma) for $3 \mathrm{hrs}$ at $4^{\circ} \mathrm{C}$. The IP product was washed 3 times with TBS-T (50 mM Tris- $\mathrm{HCl}$, pH7.4; $150 \mathrm{mM} \mathrm{NaCl}, 0.1 \%$ Tween 20) and eluted at room temperature with $0.6 \mathrm{mg}$ of the FLAG peptide. The eluate was incubated with Dynabeads Protein G conjugated with the HA monoclonal antibody 12CA5 (MSKCC Monoclonal Ab Core) for 2 hrs at $4{ }^{\circ} \mathrm{C}$. The IP product was washed 3 times with TBS-T, eluted with $0.1 \mathrm{M}$ glycine, $\mathrm{pH} 2.2$ at $56{ }^{\circ} \mathrm{C}$ followed by neutralization with $1 \mathrm{M}$ Tris $\bullet \mathrm{HCl} \mathrm{pH}$ 9.5. The final eluate was concentrated with StrataClean resin (Agilent) before extracted using 2X SDS buffer and separated in a 10\% SDS-PAGE gel (BioRAD). The gel was stained with colloidal Coomassie Brilliant Blue followed by mass spectrometry analysis (Cleveland Clinic Lerner Research Institute Proteomics and Metabolomics Core).

\section{Proteomics of Isolated Chromatin Segments (PICh)}

PICh was performed according to (47) and (79) with modifications. $5 \times 10^{10}$ WT PF cells were harvested and washed with 1 x PBS and HLB (10 mM HEPES pH 7.9; 10 mM KCl; 2.5 mM MgCl2; $1 \mathrm{mM}$ EDTA; 1 mM DTT; Complete protease (Roche), 1 mM PMSF, 1 g/ml Leupeptin, 0.5 mg/ml TLCK, and $1 \mu \mathrm{g} / \mathrm{ml}$ Pesptatin A). Cells were resuspended in HLB, incubated on ice for $10 \mathrm{~min}$, and then homogenized with 5 strokes using a glass douncer. After adding 15\% paraformaldehyde in HLB, 15-20 more strokes were performed. The lysate was centrifuged at 15,000 $\mathrm{g}$ in a swing bucket centrifuge at room temperature for $20 \mathrm{~min}$, and the pellet was resuspended in 3\% paraformaldehyde/1xPBS with rotation for $15 \mathrm{~min}$ followed by 3 washes using $1 \mathrm{x}$ PBS. The pellet was then washed with the sucrose buffer (0.3 M Sucrose; 10 mM HEPES-NaOH, pH 7.9; 1\% Triton-X100; 2 mM MgOAc), resuspended in the sucrose buffer and homogenized in a douncer with 20 strokes, then centrifuged at 15,000 g for $20 \mathrm{~min}$ at room temperature. Subsequently, the pellet was washed with 1 x PBS/0.5\% Triton X-100, resuspended in 1 x PBS/0.5\% Triton X-100/200 $\mu \mathrm{g} / \mathrm{mL}$ RNAse A with rotation for 2 hrs. After two washes with 1 x PBS and one wash with HSLB 
(10 mM HEPES-NaOH, pH 7.9; 100 mM NaCl; 2 mM EDTA; 1 mM EGTA; 0.2\% SDS; 0.1\% Sodium Sarkosyl; $1 \mathrm{mM}$ PMSF), the pellet was resuspended in HSLB and incubated at room temperature for 15 mins, followed by sonication for 13 cycles (30 sec on/off) at maximum intensity in a Bioruptor 300 (Diagenode). Samples were heated to $58^{\circ} \mathrm{C}$ for $5 \mathrm{mins}$ in a Thermomixer, then spun down at $20 \mathrm{krpm}$ for 15 minutes at room temperature. The supernatant was rotated for $2 \mathrm{hrs}$ with pre-equilibrated High Capacity Streptavidin Agarose Resin slurry (Thermo). Then the mixture was centrifuged for 2 mins and the supernatant was desalted by filtration with a Sephacryl S-400 HR column (GE Healthcare), centrifuged at $16 \mathrm{krpm}$, followed by adding SDS to a final concentration of $0.02 \%$. The resulting desalted soluble chromatin was divided into 2 equal aliquots to which either a telomere specific [TelG, 5' TTAGGGTTAGGGTTAGGGTTAGGGT 3'] or scramble (Scr, 5' GATGTGGATGTGGATGTGGATGTGG 3') LNA probe were added. Chromatin and LNA probes were hybridized in a thermocycler $\left(25^{\circ} \mathrm{C} / 3 \mathrm{~min}, 71^{\circ} \mathrm{C} / 9 \mathrm{~min}, 38^{\circ} \mathrm{C} / 60\right.$ $\min , 60^{\circ} \mathrm{C} / 3 \mathrm{~min}, 38^{\circ} \mathrm{C} / 30 \mathrm{~min}, 25^{\circ} \mathrm{C}$ final temperature), then centrifuged, immunoprecipitated, eluted, and TCA concentrated as published (79). Crosslink reversal was done by suspending the pellet in LDS NuPAGE sample buffer + 0.5 M 2-Mercaptoethanol and incubating at $99{ }^{\circ} \mathrm{C}$ for 25 mins. Protein samples were resolved in 4-12\% NuPAGE Bis-Tris gels (ThermoFisher), stained with colloidal Coomassie Brilliant Blue and stored in sealed packages until mass spectrometry analysis.

\section{EMS Clonogenic Survival Assay}

BF PolIE RNAi cells were grown with or without doxycycline for 24 hours then incubated with 2 $\mathrm{mM}$ of ethylmethanesulfonate (EMS; Sigma) or DMSO (vehicle) for two hours. Cells were washed three times with warm media and plated at a concentration of 1 cell/well in 96-well plates followed by incubation at $37^{\circ} \mathrm{C}$. Wells were checked for growth daily under the microscope until the $12^{\text {th }}$ day after plating. Survival was calculated as a percentage of EMS treated wells presenting growth in relation to the respective DMSO controls. 


\section{$\underline{\mathrm{UV} \text { and Cisplatin sensitivity tests }}$}

PolIE ${ }^{+m y c /+}$ PolIE RNAi cell were first incubated with and without doxycycline for $12 \mathrm{hrs}$ then diluted to a concentration of $1.5 \times 10^{6}$ cells $/ \mathrm{ml}$. Cells were irradiated by $0,50 \mathrm{~J} \mathrm{~m}^{-2}$, or $100 \mathrm{~J} \mathrm{~m}^{-2}$ UV using the Stratalinker ${ }^{\circledR}$ UV Crosslinker (Stratagene) or incubated with 0,10 , and $20 \mu \mathrm{M}$ cisplatin (Sigma) for $1 \mathrm{hr}$. Cells were washed free of doxycycline and cisplatin after the treatment and cell growth was monitored daily with necessary dilutions. Cell relative growth was calculated using the following formula for cells incubated with and without doxycycline: population doubling (UV/cisplatin treated)/population doubling (untreated). The average was calculated from three independent experiments.

\section{2-dimensional gel electrophoresis to detect telomeric T-circles}

Genomic DNA was isolated from PolIE RNAi cells incubated with and without doxycycline for $24 \mathrm{hrs}$ the same way as published previously (67). $5 \mu \mathrm{g}$ of MboI/AluI digested genomic DNA were separated by 2-dimensional gel electrophoresis according to (80). Specifically, DNAs were separated in the first dimension in a $0.4 \%$ agarose $1 \times$ TBE gel without ethidium bromide under 40 volts for 18 hours at room temperature. After the $1^{\text {st }}$ dimension electrophoresis, the gel was incubated in 1 x TBE/0.3 $\mu \mathrm{g} / \mathrm{ml}$ ethidium bromide for 20 minutes followed by washing with $1 \mathrm{x}$ TBE. DNA migration was verified by a long-wave UV light. DNAs were then excised from the gel, transferred to a second $1.1 \%$ agarose gel with $1 \times \mathrm{TBE} / 0.3 \mu \mathrm{g} / \mathrm{ml}$ of ethidium bromide. DNAs were electrophoresed under 150 volts at $4^{\circ} \mathrm{C}$ for $5 \mathrm{hrs}$. Subsequently, DNA was transferred to a Nylon membrane (GE Healthcare) by blotting followed by hybridization with a telomere probe.

\section{The C-circle (G-circle) Assay}

The $\varphi 29$-mediated rolling-circle assay was performed according to (60) with minor modifications. $8 \mu \mathrm{g}$ of T. brucei genomic DNA was first digested by AluI and MboI (40 U each) and $100 \mathrm{ng}$ of DNase-free RNase A, then by $\lambda$ Exonuclease and Exonuclease I to remove dsDNA. $20 \mathrm{ng}$ of the resulting DNA was incubated with $7.5 \mathrm{U} \varphi 29$ DNA polymerase (NEB) in 
reaction buffer $[1 \mu \mathrm{g} / \mu \mathrm{l}$ BSA, $0.05 \%$ Tween 20, $0.5 \mathrm{mM} \mathrm{dATP}, 0.5 \mathrm{mM}$ dGTP (or $0.5 \mathrm{mM} \mathrm{dCTP}$ for detecting G-circles) and $0.5 \mathrm{mM} \mathrm{dTTP}, 1 \times \varphi 29 \mathrm{Buffer}]$ at $30^{\circ} \mathrm{C}$ for $8 \mathrm{~h}$, then heat-inactivate the enzyme at $65^{\circ} \mathrm{C}$ for $20 \mathrm{~min}$. The reaction products were dot-blotted and UV cross-linked onto a Hybond $\mathrm{N}$ nylon membrane (GE Healthcare) followed by hybridized at $50{ }^{\circ} \mathrm{C}$ with endlabeled (CCCTAA) 4 to detect C-circles or (TTAGGG) 4 to detect G-circles.

Native in-gel hybridization to detect the telomere G-overhang structure

Genomic DNA was digested with MboI and AluI followed by the treatment with or without Exo I. Equal amount of ExoI treated and non-treated DNA were separated by agarose gel electrophoresis. The agarose gel, with separated DNA fragments inside, was dried at room temperature without denaturation. The Gel was then hybridized with an end-labeled $(\mathrm{CCCATT})_{4}$ or $(\mathrm{TTAGGG})_{4}$ probe at $50^{\circ} \mathrm{C}$. After extensive wash and exposing the hybridized gel to a phosphorimager, the gel was denatured, neutralized, and hybridized with the same oligo probe at $55^{\circ} \mathrm{C}$ followed by exposure to a phosphorimager. The hybridization signals were quantified using ImageQuant. The telomere G-overhang level was calculated by dividing the amount of native hybridization signal by the amount of post-denaturation hybridization signal.

Alternatively, DNA plugs were prepared according to (36). Intact T. brucei chromosomes were separated by pulse-field gel electrophoresis according to (42). The agarose gel, with separated chromosome DNA, was dried and hybridized with the (CCCATT) 4 or (TTAGGG) 4 probe the same way as described above. The hybridization signals were calculated the same way as described above.

Telomere Southern to estimate telomere length was performed the same way as described in (67). 


\section{EdU-labeling}

Exponentially growing BF T. brucei cells $\left(0.7-0.9 \times 10^{6}\right.$ cells $\left./ \mathrm{ml}\right)$ were incubated with $150 \mu \mathrm{M} 5$ ethynyl-2'-deoxyuridine (EdU) (Click Chemistry Tools) for 3 hrs before genomic DNA was isolated. DNA was sonicated to 400-1000 bp fragments. EdU-labeled DNA fragments were conjugated with desthiobiotin using Click chemistry regent ( $2 \mathrm{mM}$ desthiobiotin-Azide, 100 mM/500 mM CuS04/THPTA, 50 mM Na-Ascorbate, 100 mM HEPES pH 7, and 10\% DMSO). The desthiobiotin conjugated DNA was pulled-down using streptavidin beads (ThermoFisher) and eluted from the beads using biotin. The eluted DNA was dot-blotted and UV cross-linked onto a Hybond N nylon membrane (GE Healthcare) and hybridized with a telomere (or a tubulin) probe at $65^{\circ} \mathrm{C}$. The blot was exposed to a phosphorimager and the signals were quantified using ImageQuant. 


\section{Funding}

This work is supported by an NIH R01 grant AI127562 (PI, Kim), and an NIH S10 grant S10OD025252 (PI, Li). The publication cost is partly supported by GRHD at CSU.

\section{Conflict of Interest:}

The authors declare no competing interests.

\section{Acknowledgements}

We thank Dr. Keith Gull for the TAT-1 antibody. We thank the Li lab members for their comments on the manuscript. 


\section{References}

1. de Lange $\mathrm{T}$ (2005) Shelterin: the protein complex that shapes and safeguards human telomeres. Genes Dev 19(18):2100-2110.

2. $\quad$ de Lange T (2018) Shelterin-Mediated Telomere Protection. Annu Rev Genet 52:223-247.

3. Podlevsky JD, Bley CJ, Omana RV, Qi X, Chen JJ (2008) The telomerase database. Nucleic Acids Res 36(Database issue):D339-43.

4. Wynford-Thomas D, Kipling D (1997) Telomerase. Cancer and the knockout mouse. Nature 389(6651):551-552.

5. Greider CW, Blackburn EH (1987) The telomere terminal transferase of Tetrahymena is a ribonucleoprotein enzyme with two kinds of primer specificity. Cell 51(6):887-898.

6. Greider CW, Blackburn EH (1989) A telomeric sequence in the RNA of Tetrahymena telomerase required for telomere repeat synthesis. Nature 337(6205):331-337.

7. Blackburn EH (1992) Telomerases. Annu Rev Biochem 61:113-129.

8. Wellinger RJ, Wolf AJ, Zakian VA (1993) Saccharomyces telomeres acquire single-strand TG1-3 tails late in S phase. Cell 72(1):51-60.

9. McElligott R, Wellinger RJ (1997) The terminal DNA structure of mammalian chromosomes. EMBO J 16(12):3705-3714.

10. Sandhu R, Li B (2011) Examination of the telomere G-overhang structure in Trypanosoma brucei. J Vis Exp (47)

11. Sandhu R, Li B (2017) Telomerase activity is required for the telomere G-overhang structure in Trypanosoma brucei. Sci Rep 7(1):15983.

12. Griffith JD et al. (1999) Mammalian telomeres end in a large duplex loop. Cell 97(4):503514.

13. Munoz-Jordan JL, Cross GAM, de Lange T, Griffith JD (2001) t-loops at trypanosome telomeres. EMBO J 20(3):579-588.

14. Wellinger RJ, Ethier K, Labrecque P, Zakian VA (1996) Evidence for a new step in telomere maintenance. Cell 85(3):423-433.

15. Broccoli D, Smogorzewska A, Chong L, de Lange T (1997) Human telomeres contain two distinct Myb-related proteins, TRF1 and TRF2. Nat Genet 17(2):231-235. 
16. Bilaud T et al. (1997) Telomeric localization of TRF2, a novel human telobox protein. Nat Genet 17(2):236-239.

17. Wu P, Takai H, de Lange T (2012) Telomeric 3' overhangs derive from resection by Exo1 and Apollo and fill-in by POT1b-associated CST. Cell 150(1):39-52.

18. Ray S, Karamysheva Z, Wang L, Shippen DE, Price CM (2002) Interactions between telomerase and primase physically link the telomere and chromosome replication machinery. Mol Cell Biol 22(16):5859-5868.

19. Lue NF, Chan J, Wright WE, Hurwitz J (2014) The CDC13-STN1-TEN1 complex stimulates Pol $\alpha$ activity by promoting RNA priming and primase-to-polymerase switch. Nat Commun 5:5762.

20. Takai H et al. (2016) A POT1 mutation implicates defective telomere end fill-in and telomere truncations in Coats plus. Genes Dev 30(7):812-826.

21. Arnoult N, Saintome C, Ourliac-Garnier I, Riou JF, Londoño-Vallejo A (2009) Human POT1 is required for efficient telomere C-rich strand replication in the absence of WRN. Genes Dev 23(24):2915-2924.

22. Feng X et al. (2018) CTC1-STN1 terminates telomerase while STN1-TEN1 enables C-strand synthesis during telomere replication in colon cancer cells. Nat Commun 9(1):2827.

23. Gu P et al. (2018) CTC1-STN1 coordinates G- and C-strand synthesis to regulate telomere length. Aging Cell 17(4):e12783.

24. Zhang M et al. (2019) Mammalian CST averts replication failure by preventing Gquadruplex accumulation. Nucleic Acids Res 47(10):5243-5259.

25. Feng X, Hsu SJ, Kasbek C, Chaiken M, Price CM (2017) CTC1-mediated C-strand fill-in is an essential step in telomere length maintenance. Nucleic Acids Res 45(8):4281-4293.

26. Amir M et al. (2020) Structural Features of Nucleoprotein CST/Shelterin Complex Involved in the Telomere Maintenance and Its Association with Disease Mutations. Cells 9(2)

27. Wei C, Price M (2003) Protecting the terminus: t-loops and telomere end-binding proteins. Cell Mol Life Sci 60(11):2283-2294. 
28. Cross GAM, Kim HS, Wickstead B (2014) Capturing the variant surface glycoprotein repertoire (the VSGnome) of Trypanosoma brucei Lister 427. Mol Biochem Parasitol 195(1):5973.

29. Cross GAM (1975) Identification, purification and properties of clone-specific glycoprotein antigens constituting the surface coat of Trypanosoma brucei. Parasitology 71:393-417.

30. Hertz-Fowler C et al. (2008) Telomeric expression sites are highly conserved in Trypanosoma brucei. PLoS ONE 3(10):e3527.

31. Myler PJ, Allison J, Agabian N, Stuart K (1984) Antigenic variation in African trypanosomes by gene replacement or activation of alternative telomeres. Cell 39:203-211.

32. Myler P, Nelson RG, Agabian N, Stuart K (1984) Two mechanisms of expression of a variant antigen gene of Trypanosoma brucei. Nature 309:282-284.

33. Dreesen O, Li B, Cross GAM (2005) Telomere structure and shortening in telomerasedeficient Trypanosoma brucei. Nuc Acids Res 33(14):4536-4543.

34. Sandhu R et al. (2013) A trans-spliced telomerase RNA dictates telomere synthesis in Trypanosoma brucei. Cell Res 23(4):537-551.

35. Gupta SK et al. (2013) The Trypanosoma brucei telomerase RNA (TER) homologue binds core proteins of the C/D snoRNA family. FEBS Lett 587(9):1399-1404.

36. Li B, Espinal A, Cross GAM (2005) Trypanosome telomeres are protected by a homologue of mammalian TRF2. Mol Cell Biol 25(12):5011-5021.

37. Yang X, Figueiredo LM, Espinal A, Okubo E, Li B (2009) RAP1 is essential for silencing telomeric variant surface glycoprotein genes in Trypanosoma brucei. Cell 137(1):99-109.

38. Pandya UM, Sandhu R, Li B (2013) Silencing subtelomeric VSGs by Trypanosoma brucei RAP1 at the insect stage involves chromatin structure changes. Nucleic Acids Res 41(16):7673-7682.

39. Afrin M et al. (2020) TbRAP1 has an unusual duplex DNA binding activity required for its telomere localization and VSG silencing. Sci Adv 6(38):eabc4065.

40. Afrin M, Kishmiri H, Sandhu R, Rabbani MAG, Li B (2020) Trypanosoma brucei RAP1 has essential functional domains that are required for different protein interactions. $m S p h e r e$ 5(1):e00027-20. 
41. Jehi SE et al. (2014) Suppression of subtelomeric VSG switching by Trypanosoma brucei TRF requires its TTAGGG repeat-binding activity. Nucleic Acids Res 42(20):12899-12911.

42. Jehi SE, Wu F, Li B (2014) Trypanosoma brucei TIF2 suppresses VSG switching by maintaining subtelomere integrity. Cell Res 24(7):870-885.

43. Nanavaty V, Sandhu R, Jehi SE, Pandya UM, Li B (2017) Trypanosoma brucei RAP1 maintains telomere and subtelomere integrity by suppressing TERRA and telomeric RNA:DNA hybrids. Nucleic Acids Res 45(10):5785-5796.

44. Hovel-Miner GA et al. (2012) Telomere length affects the frequency and mechanism of antigenic variation in Trypanosoma brucei. PLoS Pathog 8(8):e1002900.

45. Jehi SE, Nanavaty V, Li B (2016) Trypanosoma brucei TIF2 and TRF suppress VSG switching using overlapping and independent mechanisms. PLoS One 11(6):e0156746.

46. Saha A et al. (2021) TbTRF suppresses the TERRA level and regulates the cell cycledependent TERRA foci number with a TERRA binding activity in its C-terminal Myb domain. NAR Revised manuscript submitted

47. Dejardin J, Kingston RE (2009) Purification of proteins associated with specific genomic Loci. Cell 136(1):175-186.

48. Reis H, Schwebs M, Dietz S, Janzen CJ, Butter F (2018) TelAP1 links telomere complexes with developmental expression site silencing in African trypanosomes. Nucleic Acids Res 46(6):2820-2833.

49. Leal AZ et al. (2020) Genome maintenance functions of a putative Trypanosoma brucei translesion DNA polymerase include telomere association and a role in antigenic variation. Nucleic Acids Res 48(17):9660-9680.

50. Brambati A, Barry RM, Sfeir A (2020) DNA polymerase theta (Pole)-an error-prone polymerase necessary for genome stability. Current Opinion in Genetics $\mathcal{E}$ Development 60:119-126.

51. Kent T, Mateos-Gomez PA, Sfeir A, Pomerantz RT (2016) Polymerase $\theta$ is a robust terminal transferase that oscillates between three different mechanisms during endjoining. Elife 5:e13740. 
52. Mateos-Gomez PA et al. (2015) Mammalian polymerase $\theta$ promotes alternative NHEJ and suppresses recombination. Nature 518(7538):254-257.

53. Glover L, Horn D (2012) Trypanosomal histone gammaH2A and the DNA damage response. Mol Biochem Parasitol 183(1):78-83.

54. Cestari I, McLeland-Wieser H, Stuart K (2019) Nuclear phosphatidylinositol 5phosphatase is essential for allelic exclusion of variant surface glycoprotein genes in trypanosomes. Mol Cell Biol 39(3)

55. Boothroyd CE et al. (2009) A yeast-endonuclease-generated DNA break induces antigenic switching in Trypanosoma brucei. Nature 459(7244):278-281.

56. Glover L, Alsford S, Horn D (2013) DNA break site at fragile subtelomeres determines probability and mechanism of antigenic variation in African trypanosomes. PLoS Pathog 9(3):e1003260.

57. Kim HS, Cross GAM (2010) TOPO3alpha influences antigenic variation by monitoring expression-site-associated VSG switching in Trypanosoma brucei. PLoS Pathog 6(7):e1000992.

58. Burton P, McBride DJ, Wilkes JM, Barry JD, McCulloch R (2007) Ku heterodimerindependent end joining in Trypanosoma brucei cell extracts relies upon sequence microhomology. Eukaryot Cell 6(10):1773-1781.

59. Tomaska L, Nosek J, Kramara J, Griffith JD (2009) Telomeric circles: universal players in telomere maintenance. Nat Struct Mol Biol 16(10):1010-1015.

60. Henson JD et al. (2017) The C-Circle Assay for alternative-lengthening-of-telomeres activity. Methods 114:74-84.

61. Henson JD et al. (2009) DNA C-circles are specific and quantifiable markers of alternativelengthening-of-telomeres activity. Nat Biotechnol 27(12):1181-1185.

62. Zhang JM, Zou L (2020) Alternative lengthening of telomeres: from molecular mechanisms to therapeutic outlooks. Cell Biosci 10:30.

63. Hu J et al. (2012) Antitelomerase therapy provokes ALT and mitochondrial adaptive mechanisms in cancer. Cell 148(4):651-663. 
64. Damasceno JD, Silva G, Tschudi C, Tosi LR (2017) Evidence for regulated expression of Telomeric Repeat-containing RNAs (TERRA) in parasitic trypanosomatids. Mem Inst Oswaldo Cruz 112(8):572-576.

65. Bettin N, Oss Pegorar C, Cusanelli E (2019) The Emerging Roles of TERRA in Telomere Maintenance and Genome Stability. Cells 8(3)

66. Zhang T et al. (2019) Strand break-induced replication fork collapse leads to C-circles, Coverhangs and telomeric recombination. PLoS Genet 15(2):e1007925.

67. Li B, Oestreich S, de Lange T (2000) Identification of human Rap1: implications for telomere evolution. Cell 101(5):471-483.

68. Marini F, Kim N, Schuffert A, Wood RD (2003) POLN, a nuclear PolA family DNA polymerase homologous to the DNA cross-link sensitivity protein Mus308. J Biol Chem 278(34):32014-32019.

69. Zietlow L, Smith LA, Bessho M, Bessho T (2009) Evidence for the involvement of human DNA polymerase $\mathrm{N}$ in the repair of DNA interstrand cross-links. Biochemistry 48(49):11817-11824.

70. Moldovan GL et al. (2010) DNA polymerase POLN participates in cross-link repair and homologous recombination. Mol Cell Biol 30(4):1088-1096.

71. Yoon JH et al. (2019) Error-Prone Replication through UV Lesions by DNA Polymerase $\theta$ Protects against Skin Cancers. Cell 176(6):1295-1309.e15.

72. Feng W et al. (2019) Genetic determinants of cellular addiction to DNA polymerase theta. Nat Commun 10(1):4286.

73. Shima N et al. (2003) Phenotype-based identification of mouse chromosome instability mutants. Genetics 163(3):1031-1040.

74. Cross M, Taylor MC, Borst P (1998) Frequent loss of the active site during variant surface glycoprotein expression site switching in vitro in Trypanosoma brucei. Mol Cell Biol 18(1):198-205.

75. Kim HS, Cross GAM (2011) Identification of Trypanosoma brucei RMI1/BLAP75 homologue and its roles in antigenic variation. PLoS One 6(9):e25313. 
76. Robinson NP, Burman N, Melville SE, Barry JD (1999) Predominance of duplicative VSG gene conversion in antigenic variation in African trypanosomes. Mol Cell Biol 19(9):5839_ 5846.

77. Wirtz E, Leal S, Ochatt C, Cross GAM (1999) A tightly regulated inducible expression system for dominant negative approaches in Trypanosoma brucei. Mol Biochem Parasitol 99(1):89-101.

78. Wang Z, Morris JC, Drew ME, Englund PT (2000) Inhibition of Trypanosoma brucei gene expression by RNA interference using an integratable vector with opposing T7 promoters. J Biol Chem 275(51):40174-40179.

79. Antao JM, Mason JM, Dejardin J, Kingston RE (2012) Protein landscape at Drosophila melanogaster telomere-associated sequence repeats. Mol Cell Biol 32(12):2170-2182.

80. Cohen S, Mechali M (2002) Formation of extrachromosomal circles from telomeric DNA in Xenopus laevis. EMBO Rep 3(12):1168-1174.

81. Woods A et al. (1989) Definition of individual components within the cytoskeleton of Trypanosoma brucei by a library of monoclonal antibodies. J Cell Sci 93 (Pt 3):491-500. 


\section{Figure Legends}

Figure 1. PolIE interacts with TbTRF and TbTIF2. (A) Venn diagram showing the number of protein candidates identified in IP of the TbTRF/TbTIF2 protein complex and in PICh. (B) Western blotting showing the expression of TbTIF-F2H and PolIE-myc in the indicated strains. (C) and (D) IP using the myc Ab 9E10 (MSKCC Antibody \& Bioresource Core Facility) (C) and a TbTRF rabbit $\mathrm{Ab}(36)(\mathrm{D})$ and IgG (as a negative control) in TbTIF2 ${ }^{+\mathrm{F} 2 \mathrm{H} /+}$ POLIE ${ }^{+m y c /+}$ cells. Western analyses were performed using the myc Ab 9E10, the HA antibody (HA probe, Santa Cruz Biotechnologies), and a TbTRF chicken Ab (37).

Figure 2. PollE is enriched at the telomere. (A) ChIP using the myc Ab 9E10, a TbTRF rabbit antibody (36), and IgG (as a negative control) in POLIE ${ }^{\text {myc/t }}$ cells. The ChIP products were detected in Southern slot blots using a telomere and a tubulin probe. (B) Quantification results of PolIE-myc and TbTRF ChIP in POLIE ${ }^{+m y c /+}$ cells. Average enrichment (ChIP/Input) was calculated from three or four independent experiments. (C) IF in POLIE ${ }^{+m y c /+}$ cells using the myc Ab 9E10 and a TbTRF rabbit Ab. DNA was stained with DAPI. All panels are of the same scale and the size bar is shown in one panel. Representative cells in various cell cycle stages are shown. (D) Western blotting showing depletion of TbTRF in POLIE ${ }^{+m y c /+}$ TbTRF RNAi cells after a 24-hr induction. A TbTRF rabbit Ab, the myc Ab 9E10, and the tubulin Ab TAT-1 (81) were used. (E) Quantification results of PolIE-myc and TbTRF ChIP in POLIE ${ }^{+ \text {myc/+ }}$ TbTRF RNAi cells before (-Dox) and after (+Dox) the induction of TbTRF RNAi. Average enrichment (ChIP/Input) was calculated from three independent experiments. In this and other figures, error bars represent standard variation. $P$ values of unpaired $t$-tests are shown in $(\mathrm{B})$ and $(\mathrm{E})$.

Figure 3. PolIE-depleted cells are hypersensitive to cisplatin treatment and UV irradiation. (A) Western blotting showing depletion of PolIE-myc in POLIE ${ }^{\text {myc/+ }}$ RNAi cells. The myc Ab 9E10 and the tubulin Ab TAT-1 (81) were used. (B) Growth curves of POLIE ${ }^{+m y c /+}$ RNAi cells before (Dox) and after (+Dox) the induction of RNAi. (C) POLIE ${ }^{+m y c /+}$ RNAi cells incubated with or without doxycycline for $12 \mathrm{hrs}$ were treated with and without $20 \mu \mathrm{M}$ cisplatin for $1 \mathrm{hr}$ before 
cells were washed free of doxycycline and cisplatin. Subsequently, cell growth was monitored (left) and relative growth (treated/untreated) was calculated from three independent experiments and shown on the right. (D) POLIE ${ }^{+m y c /+}$ RNAi cells incubated with or without doxycycline for $12 \mathrm{hrs}$ were irradiated with and without $50 \mathrm{~mJ}$ and $100 \mathrm{~mJ} \mathrm{UV}$ before cells were washed free of doxycycline. Subsequently, cell growth was monitored (left) and relative growth (treated/untreated) was calculated from three independent experiments and shown on the right. Asterisks indicate significant difference between relative growth of uninduced and induced cells.

Figure 4. Depletion of PolIE leads to an increased amount of DNA damage. (A) Depletion of PolIE-myc in POLIE ${ }^{+m y c /+}$ RNAi cells did not change TbTRF or TbRAP1 protein levels but increased the $\gamma \mathrm{H} 2 \mathrm{~A}$ level. The myc Ab 9E10, a TbTRF rabbit Ab (36), a TbRAP1 rabbit Ab (37), a $\gamma \mathrm{H} 2 \mathrm{~A}$ rabbit $\mathrm{Ab}(43)$, and the tubulin Ab TAT-1 (81) were used in western analyses. (B) Quantification of percent of $P O L I E^{\text {myc/t+ }}$ RNAi cells that are positive for the $\gamma \mathrm{H} 2 \mathrm{~A}$ signal in IF before and after the induction of PolIE RNAi for 24 hrs. (C) Representative Southern slot blots of $\gamma \mathrm{H} 2 \mathrm{~A}$ ChIP products. ChIP was performed in POLIE ${ }^{+m y c /+}$ RNAi cells before and 24 hrs after induction of PolIE RNAi using a $\gamma \mathrm{H} 2 \mathrm{~A}$ rabbit $\mathrm{Ab}$ (43) and IgG (as a negative control). (D) Quantification of Southern blotting results of $\gamma \mathrm{H} 2 \mathrm{~A}$ ChIP products. The average enrichment of $\gamma \mathrm{H} 2 \mathrm{~A}$ at the telomere (ChIP/Input) was calculated from five independent experiments. (E) The $\gamma \mathrm{H} 2 \mathrm{~A}$ ChIP products were analyzed by quantitative PCR using primers specific to the active VSG2, silent VSG16 and $m$ VSG531, 70 bp repeats in the active ES (70 bp BES) and a silent ES (70 bp telo), and chromosome internal SNAP50 and rDNA. The average enrichment of $\gamma \mathrm{H} 2 \mathrm{~A}$ at each indicated locus was calculated from five to seven independent experiments. $P$ values of unpaired $t$-tests are shown in (B), (D), and (E).

Figure 5. A transient depletion of PolIE leads to an increased VSG switching rate. (A) Growth curves of S/IEi cells under several conditions: without induction (-Dox), continued induction 
(+Dox), a transient induction (+Dox for $30 \mathrm{hrs}$ followed by wash), and re-induction (after a transient 30-hr induction and wash, +Dox at 96 hrs). (B) Top, inducing PolIE RNAi for $30 \mathrm{hrs}$ resulted in a transient depletion of PolIE-myc in S/IEi/PolIE-myc cells. Bottom, induced ectopic PolIE-myc expression in S/IEi+ecPolIE-myc cells. The myc Ab 9E10 and a tubulin Ab TAT-1 (81) were used in western blotting. (C) VSG switching rates in the indicated strains. $P$ values of unpaired $t$-tests are shown. (D) Percent of various VSG switching mechanisms in the indicated strains. The total number of switchers characterized in each strain is listed on top of each column.

Figure 6. Depletion of PolIE leads to an increased amount of telomere T-circles and C-circles. (A) 2D gel electrophoresis of AluI/Mbol digested genomic DNA isolated from POLIE ${ }^{+m y c /+}$ RNAi cells before (-Dox) and after (+Dox) 24-hr of RNAi induction. (B) Diagram showing expected migration patterns of linear and circular DNAs in 2D electrophoresis. (C) Quantification of Southern results after 2D electrophoresis. The average T-circle amount (percent of total telomeric DNA) was calculated from six independent experiments. (D) The C-circle products from indicated cells were detected in Southern slot blotting using either a (CCCTAA) 4 (TelC) probe or a (TTAGGG) 4 (TelG) probe. (E) Quantification of the telomeric C-circle amount in the C-circle assay. The C-circle level in WT cells was arbitrarily set to 1, and relative C-circle levels in other cells were quantified using the WT level as a reference. The average C-circle signal level was calculated from three or four independent experiments. $P$ values of unpaired $t$-tests are shown in $(C)$ and $(E)$.

Figure 7. PolIE Depletion decreases the TERRA level. (A) Northern hybridization detecting TERRA in PollE RNAi cells before (-Dox) and after (+Dox) the RNAi induction. The telomerase RNA component TbTR was detected as a loading control. (B) Top, a representative northern slot blot detecting TERRA in PolIE RNAi cells. Tubulin RNA was detected as a loading control. Bottom, quantification of relative TERRA levels in PolIE RNAi cells (normalized against the 
TbTR level). Average was calculated from three independent northern slot blot experiments. The $p$ value from the unpaired $t$-test is shown.

Figure 8. PollE-depletion resulted in an increased level of the telomere G-overhang. Genomic DNAs were isolated from WT and POLIE ${ }^{+m y c /+}$ RNAi (labeled as PolIE RNAi) cells (A \& B) or from $T_{b T R^{-/}}$PollE RNAi cells (D) before (-Dox) and after (+Dox) a 24-hr induction of RNAi. The genomic DNA was treated with and without ExoI (NEB), which is a 3' single-strand DNAspecific exonuclease. In-gel hybridization was performed using a (CCCTAA) 4 (TelC) probe or a (TTAGGG)4 (TelG) probe (listed below the hybridization images) first under the native condition (left) and then after denaturation and neutralization (right). (C \& E) Quantification of the relative G-overhang level (using that in WT cells as a reference) in POLIE ${ }^{\text {+myc/+ }}$ RNAi cells (C) and in TbTR ${ }^{--}$PolIE RNAi cells (E) before (-Dox) and after (+Dox) PolIE RNAi induction. The average telomere G-overhang level was calculated from three to five independent experiments. $P$ values of the unpaired $t$-test are shown in $(C)$ and $(E)$.

Figure 9. (A) Depletion of PollE did not affect the bulk telomere length within a short time frame. Genomic DNA isolated from POLIE ${ }^{+m y c /+}$ RNAi cells before $(0 \mathrm{hr})$ and after $(24 \mathrm{hr})$ inducing PolIE RNAi were digested with AluI and MboI and separated by agarose gel electrophoresis. Southern blotting was performed using a telomere probe. (B) and (C) In POLIE ${ }^{\text {myc/+ }}$ RNAi (B) or TbTR ${ }^{-/}$PolIE RNAi (C) cells, before (-Dox) and after 12-hr (+Dox) of PolIE RNAi induction, EdU-labeled nascent DNA was conjugated with desthiobiotin and pulled-down by streptavidin beads followed by Southern blotting using a telomere and a tubulin probe. The fold changes in the amount of EdU-labeled telomeric and tubulin DNA (+Dox/-Dox) were quantified and shown at the bottom in (B) and (C). The average change was calculated from five independent experiments. $P$ values of unpaired $t$-tests are shown. 
Tonini et al. Figure 1

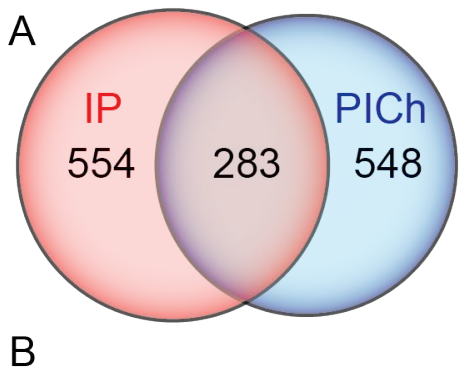

TbTIF2 ${ }^{+\mathrm{F} 2 \mathrm{H} /+}$

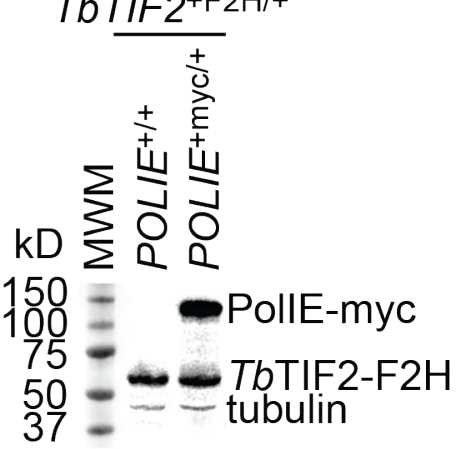

C

IP

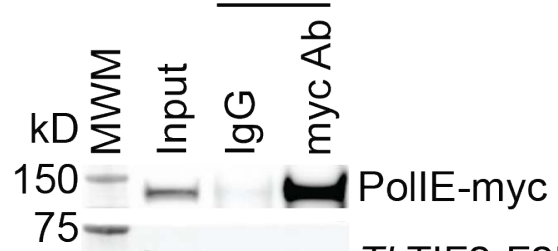

TbTIF2-F2H

50

37

D

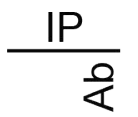

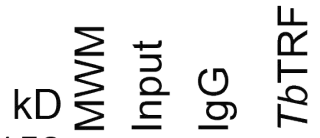

150- $\longrightarrow$ PollE-myc

TbTIF2-F2H $37 \div=\quad \Longrightarrow$ TbTRF 
Tonini et al. Figure 2

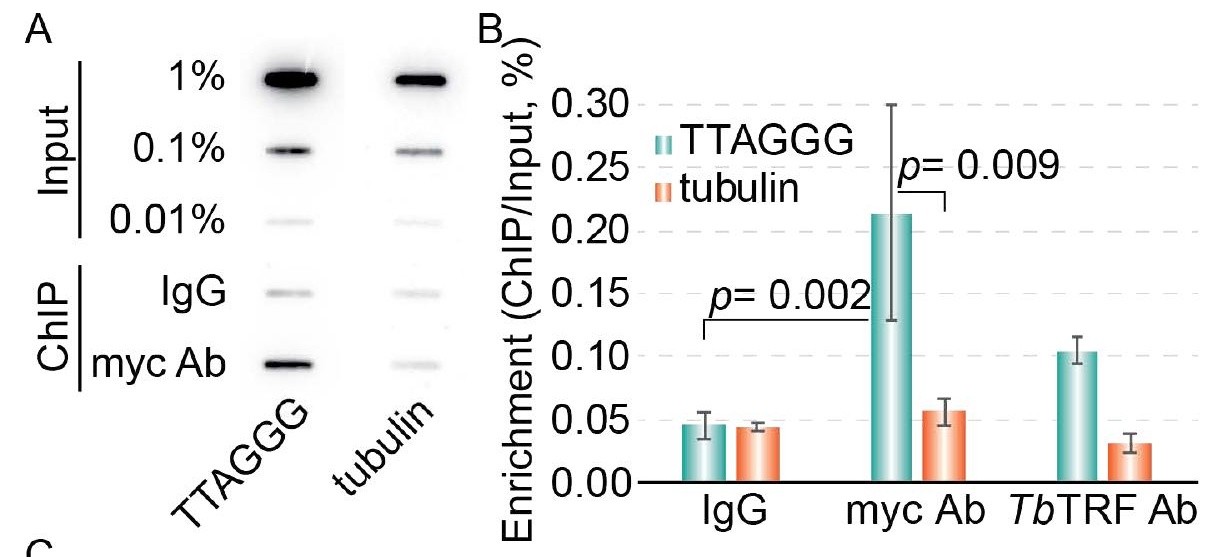

C
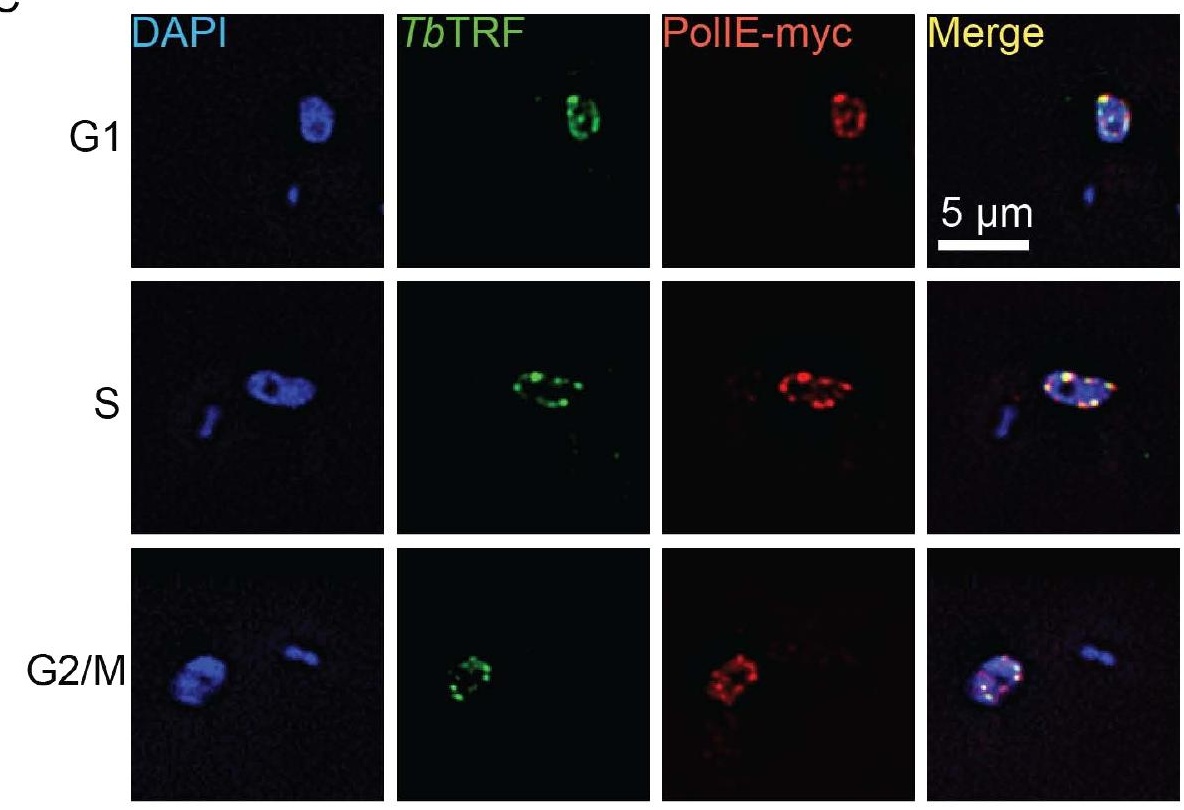

P
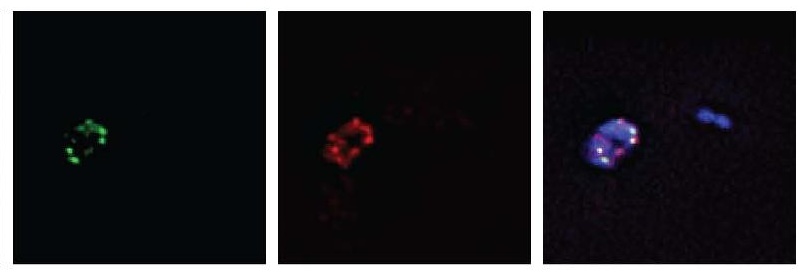

post M
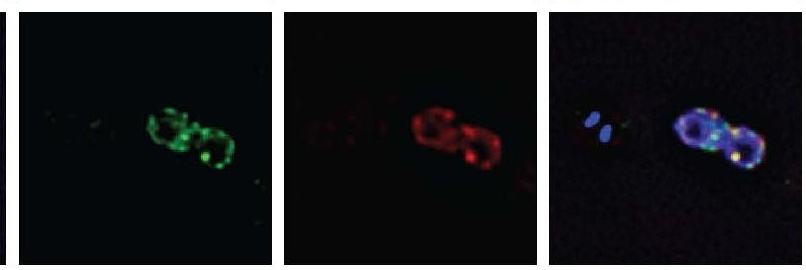

POLIE ${ }^{+m y c /+}$

TbTRF RNAi

kD 024 hrs + Dox

50-

TbTRF

150- $=$ PollE-myc

100

$\mathrm{E}$

TTAGGG

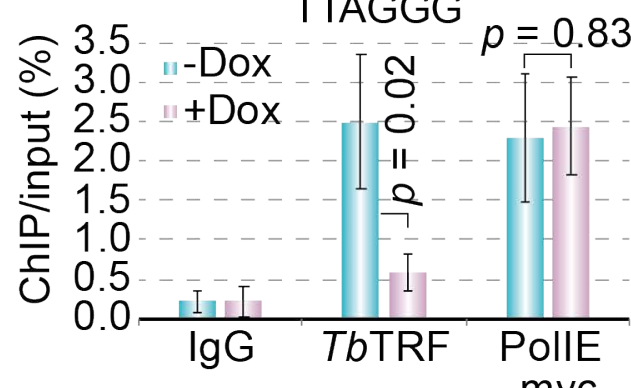


Tonini et al. Figure 3

A

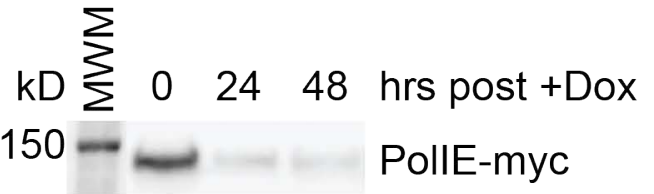

$50-\longrightarrow$ tubulin
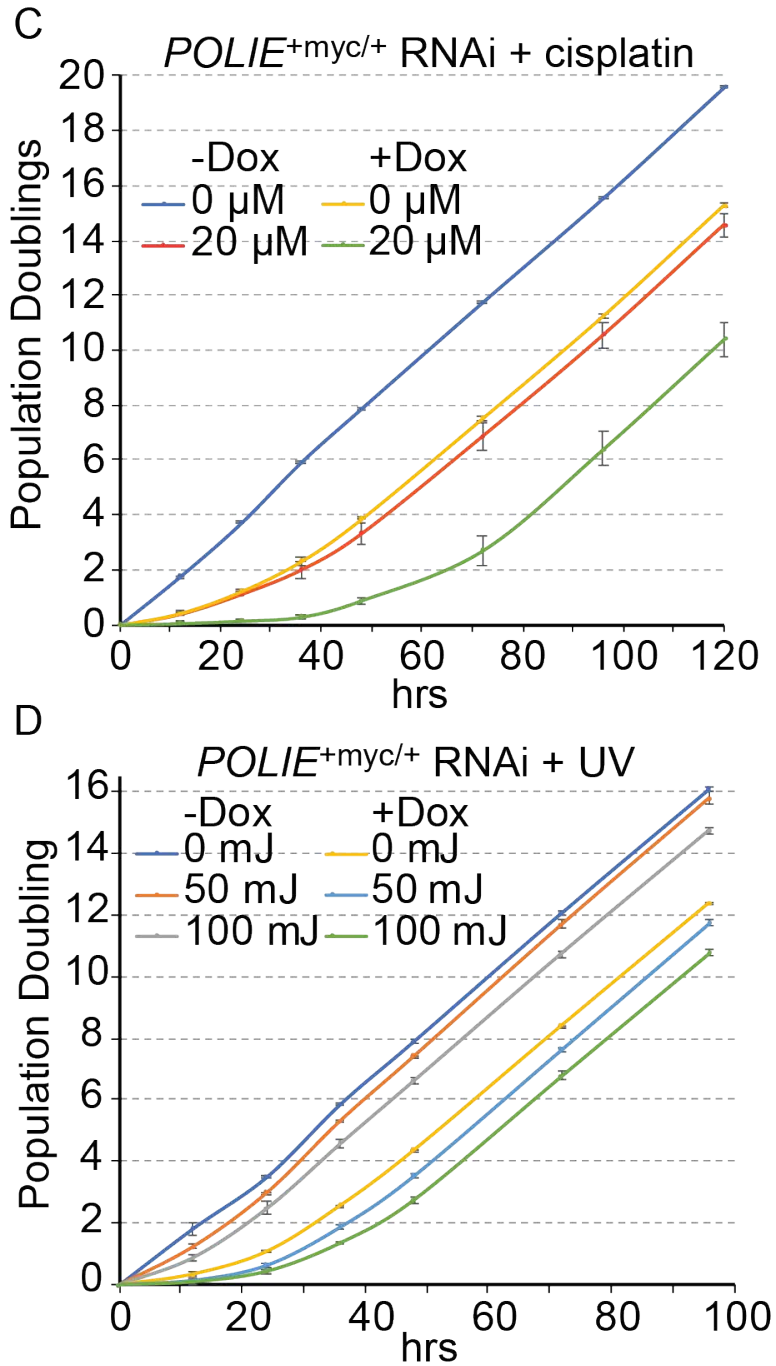
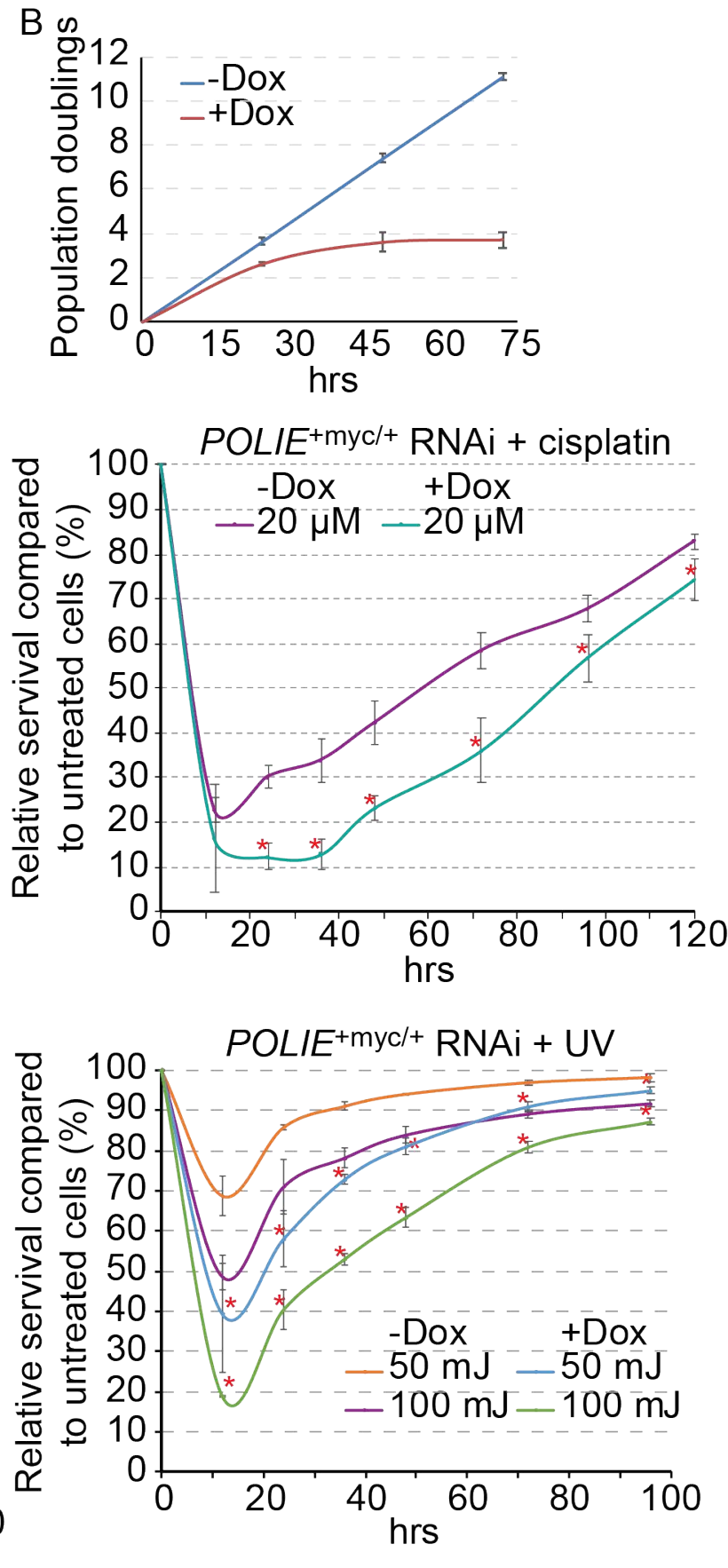
Tonini et al. Figure 4

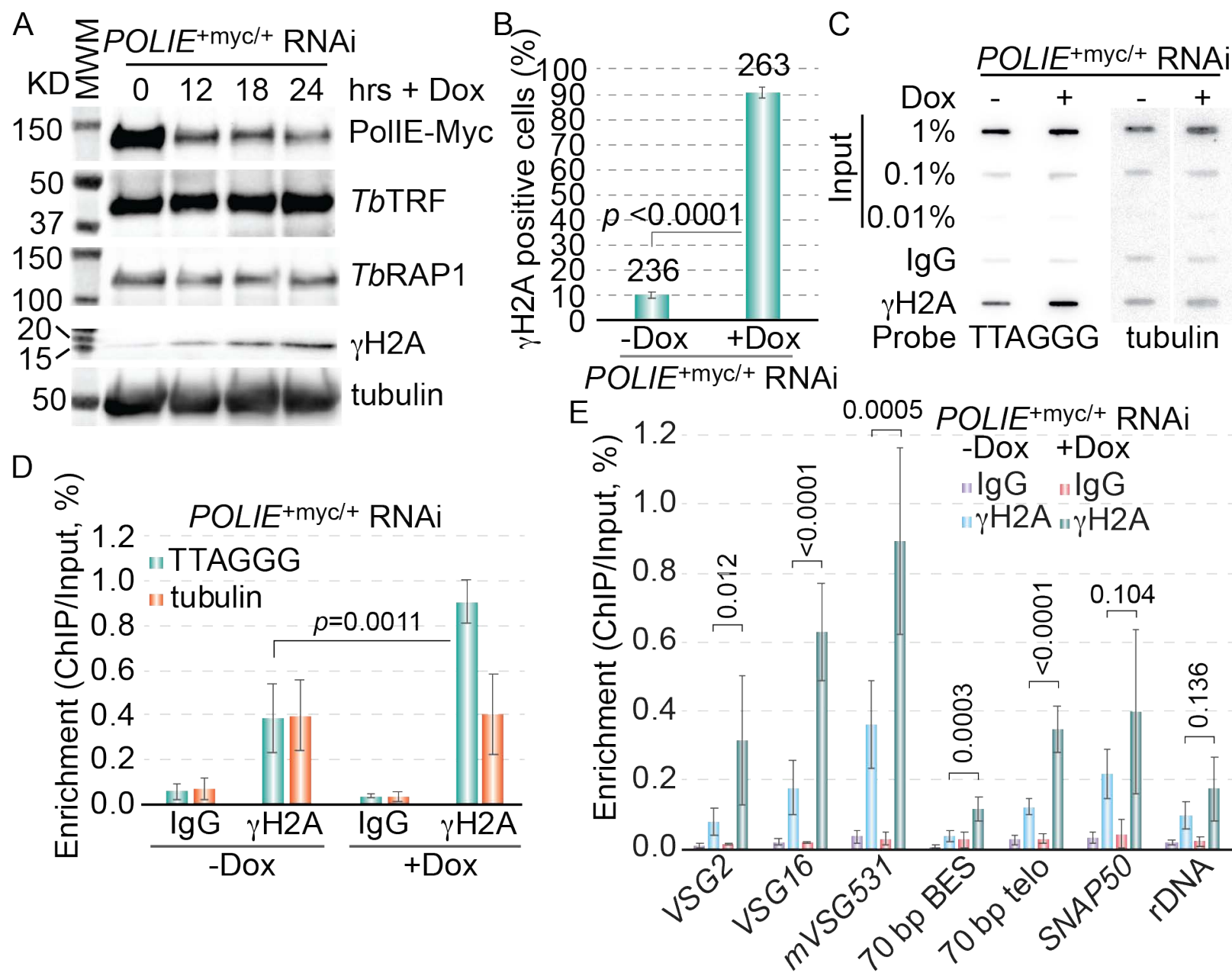


Tonini et al. Figure 5
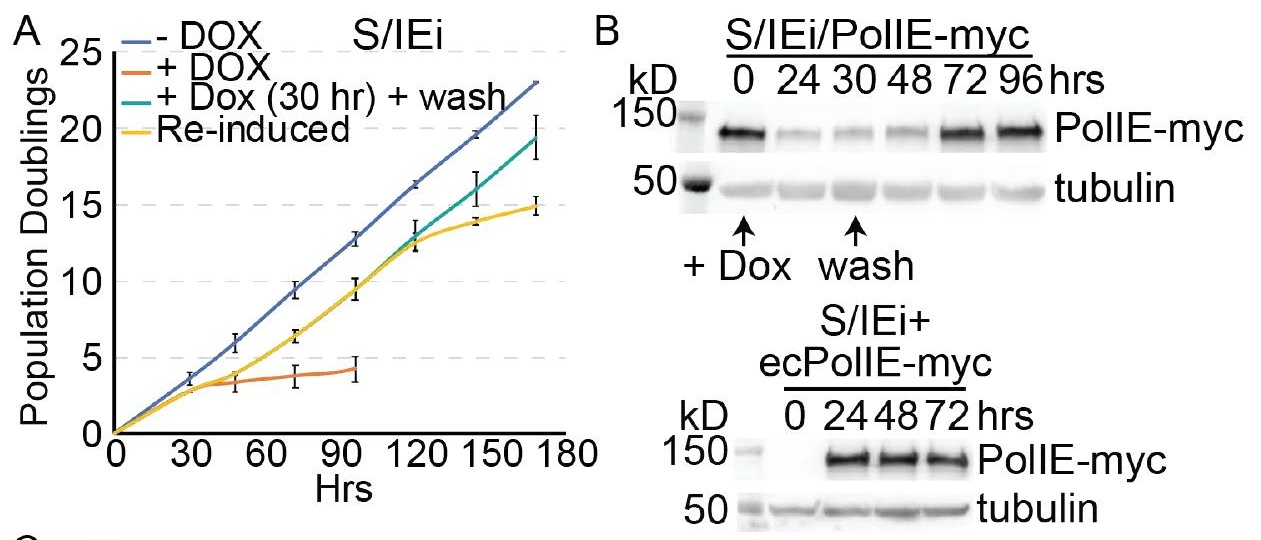

C

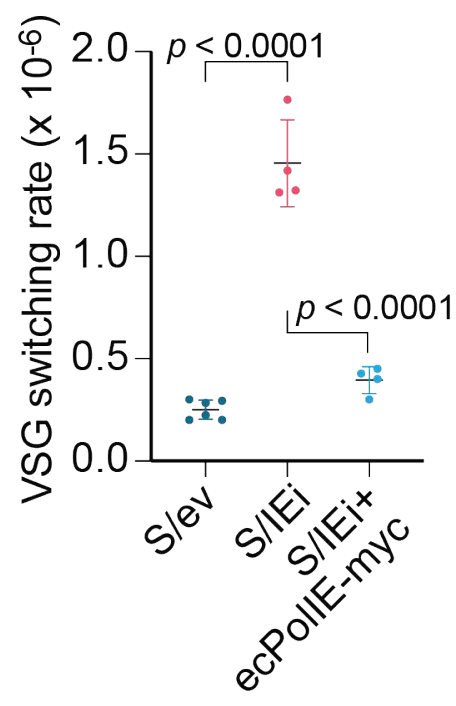

D

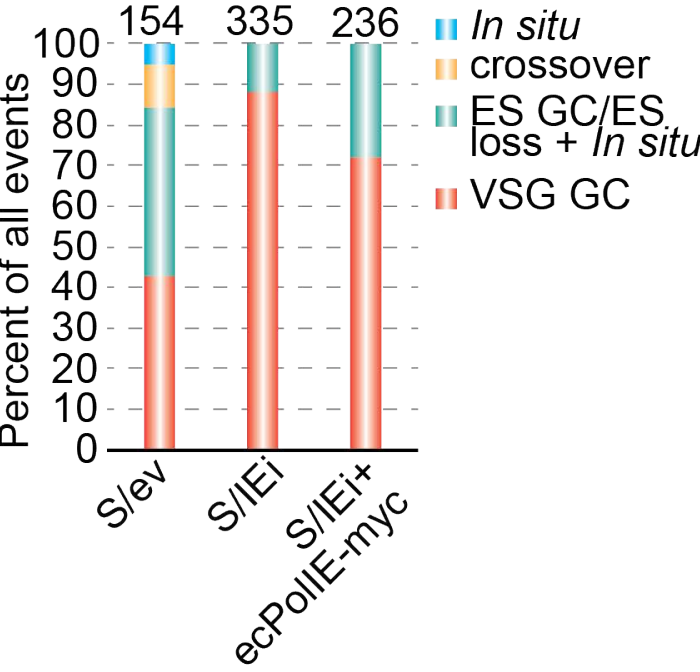


A

\begin{tabular}{ll}
\multicolumn{3}{c}{ POLIE ${ }^{+m y c /+}$ RNAi } \\
\hline -Dox
\end{tabular}

$\longrightarrow$ 1st dimension

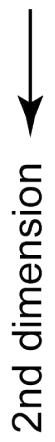

B

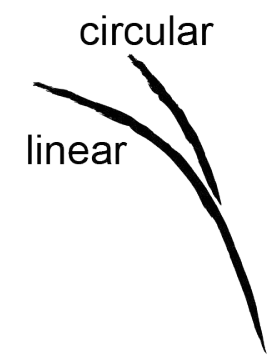

C

POLIE ${ }^{+m y c /+}$ RNAi

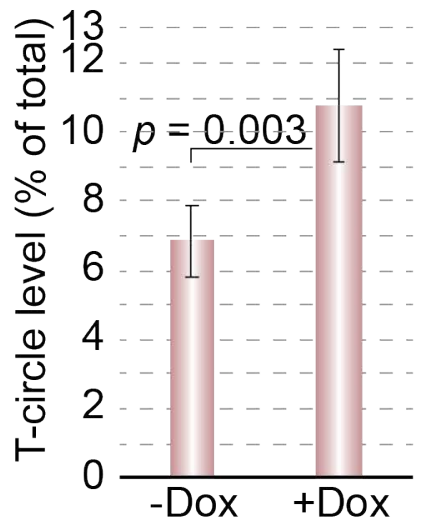

D $\quad \phi 29 \mathrm{Pol} \quad-\quad+\quad+\quad+\quad+\quad E$

No DNA

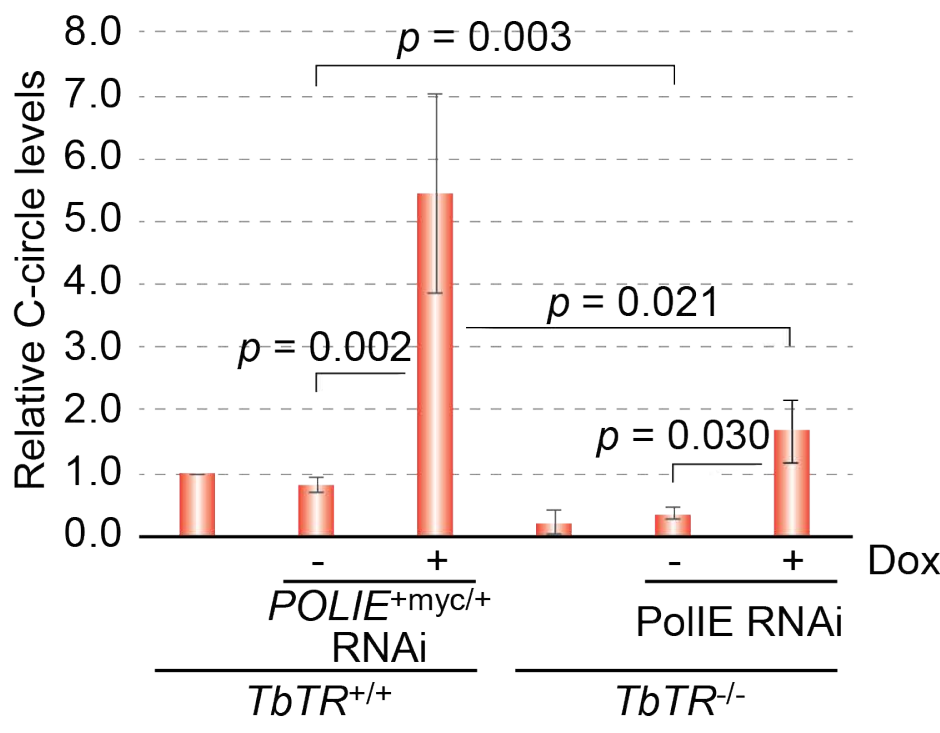

TelG 


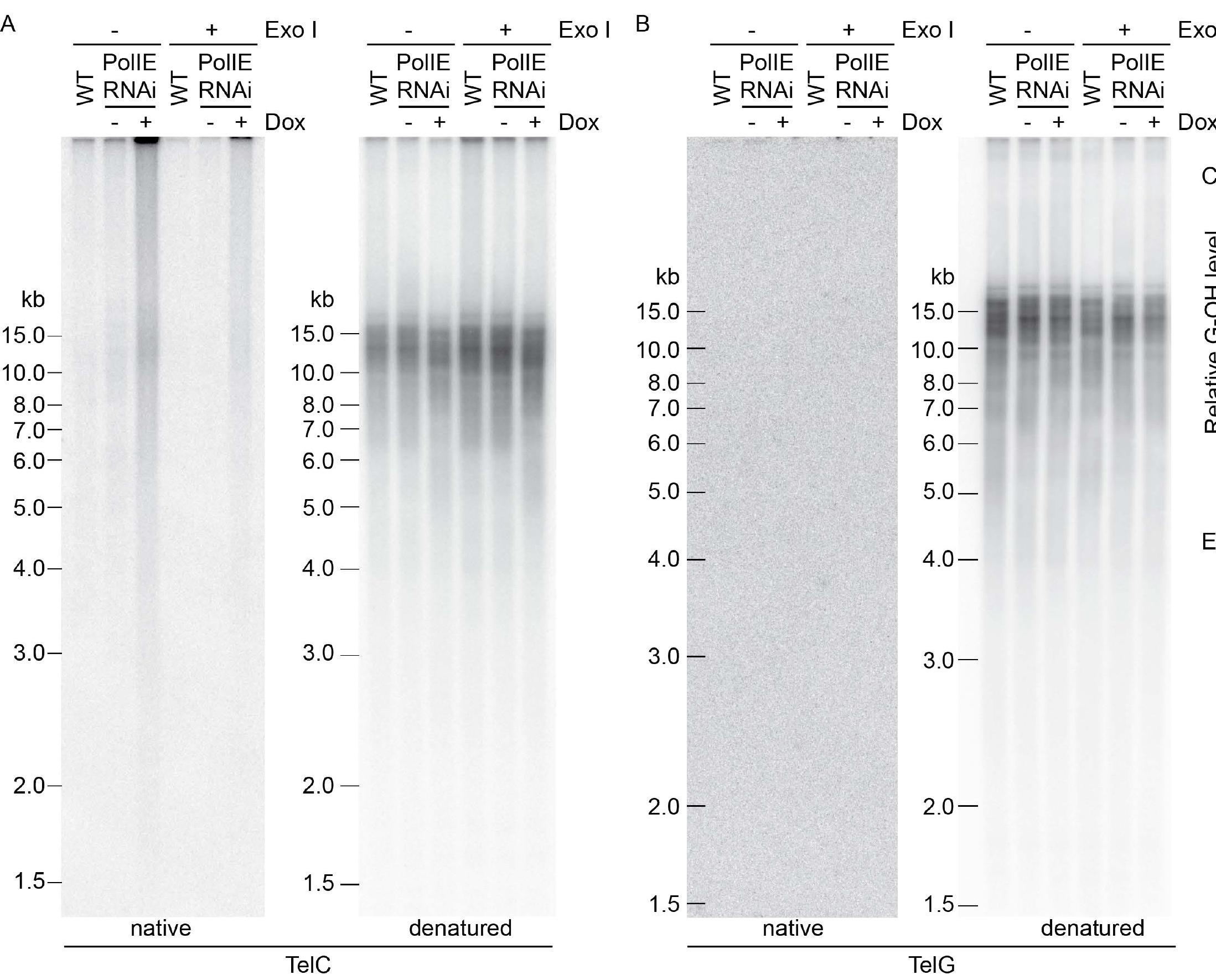

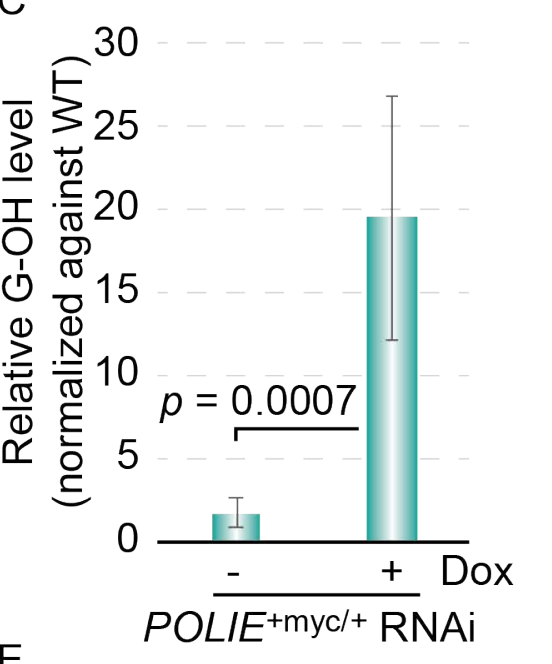

\begin{tabular}{c} 
TbTR ${ }^{-/-}$ \\
PollE RNAi \\
\hline-+-+ Dox
\end{tabular}

$\mathrm{E}$

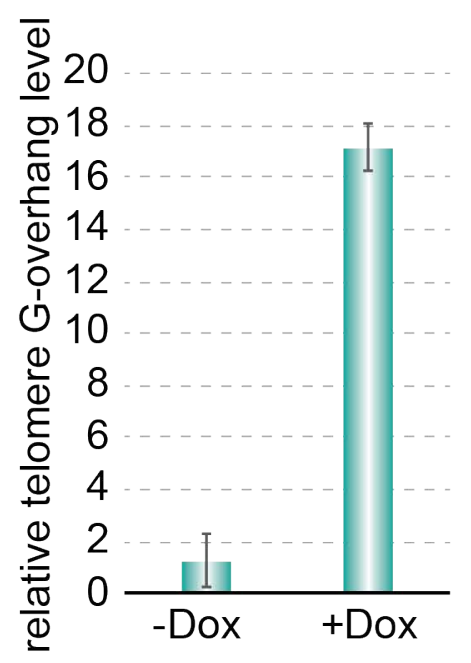

native denatured
TelC

native denatured
TelC

$T_{b T R^{-/-}}$

PollE RNAi - + Rox

-+-+ Dox

native denatured 
Tonini et al. Figure 9

A POLIE ${ }^{+m y c /+}$ RNAi

$\begin{array}{lllll}0 & 24 & 0 & 24 & \text { hrs post +Dox }\end{array}$

\section{$\mathrm{Kb}$}

15

$10-$

$8-$

7

$6-$

$5-$

4

3
B

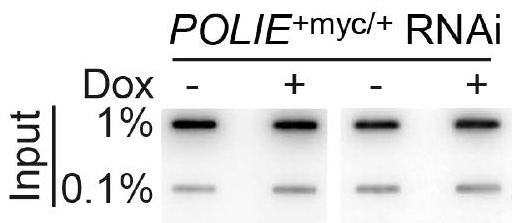

Pull-Down

Probe TTAGGG tubulin

- TTAGGG

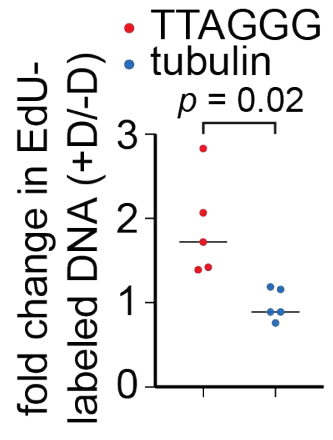

C

TbTR ${ }^{-/-}$PollE RNAi

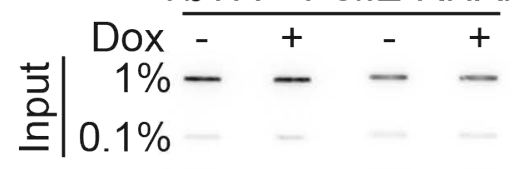

Pull-Down - - - -

Probe TTAGGG tubulin

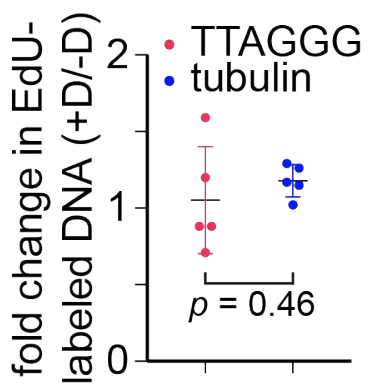

